\title{
Guidelines for the Diagnosis and Treatment of Hepatocellular Carcinoma (2019 Edition)
}

\author{
Jian Zhou ${ }^{a} \quad$ Huichuan Sun ${ }^{a}$ Zheng Wang ${ }^{a}$ Wenming Cong ${ }^{b}$ Jianhua Wang ${ }^{c}$ \\ Mengsu Zeng ${ }^{d}$ Weiping Zhou ${ }^{e}$ Ping Bie ${ }^{f}$ Lianxin Liug Tianfu Wen ${ }^{\text {h }}$ Guohong Han ${ }^{\mathrm{i}}$ \\ Maoqiang Wang ${ }^{j}$ Ruibao Liu ${ }^{k}$ Ligong Lu' Zhengang Ren ${ }^{a}$ Minshan Chen $^{m}$ \\ Zhaochong Zeng $^{\mathrm{n}}$ Ping Liang $^{\circ}$ Changhong Liang $^{p}$ Min Chen $^{q}$ Fuhua Yan $^{r}$ \\ Wenping Wang ${ }^{\mathrm{S}}$ Yuan Ji ${ }^{\mathrm{t}}$ Jingping Yun ${ }^{\mathrm{u}}$ Dingfang Cai ${ }^{\mathrm{V}}$ Yongjun Chen ${ }^{\mathrm{w}}$ \\ Wenwu Cheng ${ }^{\mathrm{x}}$ Shuqun Cheng ${ }^{\mathrm{A}}$ Chaoliu Dai ${ }^{\mathrm{y}}$ Wenzhi Guo ${ }^{z}$ Baojin Hua ${ }^{\mathrm{A}}$ \\ Xiaowu Huang ${ }^{\mathrm{a}}$ Weidong $\mathrm{Jia}^{\mathrm{B}}$ Yaming $\mathrm{Li}^{\mathrm{C}}$ Yexiong $\mathrm{Li}^{\mathrm{D}}$ Jun Liang ${ }^{\mathrm{E}}$ Tianshu Liu ${ }^{\mathrm{F}}$ \\ Guoyue Lv ${ }^{G}$ Yilei Mao ${ }^{H}$ Tao Pengl Weixin Ren' Hongcheng Shi ${ }^{\mathrm{K}}$ Guoming Shi ${ }^{\mathrm{a}}$ \\ Kaishan Tao ${ }^{\mathrm{L}}$ Wentao Wang ${ }^{\mathrm{M}}$ Xiaoying Wang ${ }^{\text {a }}$ Zhiming Wang $^{\mathrm{N}}$ Bangde Xiang $^{\mathrm{O}}$ \\ Baocai Xing ${ }^{\mathrm{P}}$ Jianming $\mathrm{Xu}^{\mathrm{Q}}$ Jiamei Yang ${ }^{\mathrm{e}}$ Jianyong Yang ${ }^{\mathrm{R}}$ Yefa Yang ${ }^{\mathrm{S}}$ Yunke Yang ${ }^{\mathrm{V}}$ \\ Shenglong Ye ${ }^{a}$ Zhengyu Yin ${ }^{\top}$ Bixiang Zhang ${ }^{U}$ Boheng Zhang ${ }^{a}$ Leida Zhang ${ }^{\vee}$ \\ Shuijun Zhang ${ }^{W}$ Ti Zhang ${ }^{X}$ Yongfu ZhaoW Honggang Zheng ${ }^{Y}$ Jiye Zhu ${ }^{Z}$ \\ Kangshun Zhu ${ }^{\alpha}$ Rong Liu ${ }^{c}$ Yinghong Shi ${ }^{a}$ Yongsheng Xiao ${ }^{a}$ Zhi Dai $^{a}$ Gaojun Teng ${ }^{\beta}$ \\ Jianqiang Cai ${ }^{\gamma}$ Weilin Wang ${ }^{\delta} \quad$ Xiujun Cai $^{\varepsilon} \quad$ Qiang Li ${ }^{X} \quad$ Feng Shen $^{\mathrm{e}}$ Shukui Qin ${ }^{\zeta}$ \\ Jiahong Dong ${ }^{\ominus} \quad$ Jia Fan $^{\mathrm{a}}$
}

\begin{abstract}
aLiver Cancer Institute, Zhongshan Hospital, Fudan University, Shanghai, China; bepartment of Pathology, The Eastern Hepatobiliary Surgery Hospital, Second Military Medical University, Shanghai, China; 'Department of Interventional Radiology, Zhongshan Hospital, Fudan University, Shanghai, China; ${ }^{\mathrm{d} D e p a r t m e n t}$ of Radiology, Zhongshan Hospital, Fudan University, Shanghai,

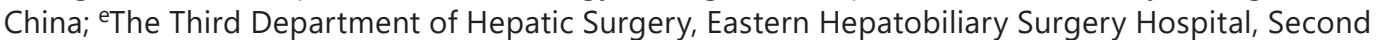
Military Medical University, Shanghai, China; ${ }^{\mathrm{I}}$ Institute of Hepatobiliary Surgery, Southwest Hospital, Third Military Medical University, Chongqing, China; ${ }^{9}$ Department of General Surgery, The First Affiliated Hospital of Harbin Medical University, Harbin, China; hepartment of Liver Surgery, West China Hospital of Sichuan University, Chengdu, China; 'Department of Liver Diseases and Digestive Interventional Radiology, Xijing Hospital, Fourth Military Medical University, Xi'an, China; jDepartment of Interventional Radiology, Chinese PLA General Hospital, Beijing, China; ${ }^{\text {kDepartment }}$ of Interventional Radiology, The Tumor Hospital of Harbin Medical University, Harbin, China; 'Department of Interventional Oncology, Guangdong General Hospital, Guangdong Academy of Medical Sciences, Guangzhou, China; mDepartment of Hepatobiliary Surgery, Sun Yat-sen University Cancer Center, Guangzhou, China; " Department of Radiation Oncology, Zhongshan Hospital, Fudan University, Shanghai, China; ${ }^{\circ}$ Department of Interventional Ultrasound, Chinese PLA General Hospital,

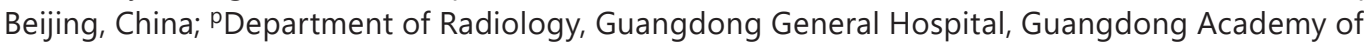
Medical Sciences, Guangzhou, China; 'Editorial Department of Chinese Journal of Digestive Surgery, Chongqing, China; 'Department of Radiology, Ruijin Hospital Affiliated to Shanghai Jiaotong University School of Medicine, Shanghai, China; 'Department of Ultrasound, Zhongshan Hospital, Fudan University, Shanghai, China; 'Department of Pathology, Zhongshan Hospital, Fudan University, Shanghai, China; "Department of Pathology, Tumor Prevention and Treatment Center, Sun Yat-sen University, Guangzhou, China;
\end{abstract}

Jian Zhou, Huichuan Sun, and Zheng Wang contributed equally to this study. 
'Department of Integrative Medicine, Zhongshan Hospital, Fudan University, Shanghai, China; wDepartment of Hematology, Ruijin Hospital North, Shanghai Jiao Tong University School of Medicine, Shanghai, China; ${ }^{x}$ Department of Integrated Therapy, Fudan University Shanghai Cancer

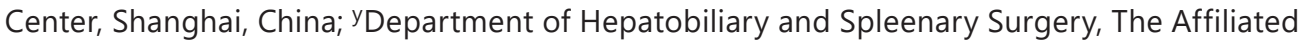
Shengjing Hospital, China Medical University, Shenyang, China; ' Department of Hepatobiliary and Pancreatic Surgery, The First Affiliated Hospital of Zhengzhou University, Zhengzhou, China; ${ }^{A}$ Graduate School, Beijing University of Chinese Medicine, Beijing, China; ${ }^{B}$ Department of Hepatic Surgery, Affiliated Provincial Hospital, Anhui Medical University, Hefei, China; ${ }^{C}$ Department of Nuclear Medicine, The First Hospital of China Medical University, Shenyang, China; ${ }^{D}$ Department of Radiation Oncology, National Cancer Center/Cancer Hospital, Chinese Academy of Medical Sciences and Peking Union Medical College, Beijing, China; EDepartment of Oncology, Peking University International Hospital, Beijing, China; F Department of Oncology, Zhongshan Hospital, Fudan University, Shanghai, China; ${ }^{G}$ Department of General Surgery, The First Hospital of Jilin University, Jilin, China; ${ }^{H}$ Department of Liver Surgery, Peking Union Medical College (PUMC) Hospital, PUMC and Chinese Academy of Medical Sciences, Beijing, China; 'Department of Hepatobiliary Surgery, The First Affiliated Hospital of Guangxi Medical University, Nanning, China; 'Department of Interventional Radiology The First Affiliated Hospital of Xinjiang Medical University, Urumqi, China; ${ }^{K}$ Department of Nuclear Medicine, Zhongshan Hospital, Fudan University, Shanghai, China; 'Department of Hepatobiliary Surgery, Xijing Hospital, Fourth Military Medical University, Xi'an, China; MDepartment of Liver Surgery, West China Hospital of Sichuan University, Chengdu, China; ${ }^{N}$ Department of Infectious Diseases, Xiangya Hospital, Central South University, Changsha, China; ${ }^{\circ}$ Department of Hepatobiliary Surgery, Affiliated Tumor Hospital of Guangxi Medical University, Nanning, China; P Department of Hepato-PancreatoBiliary Surgery, Peking University Cancer Hospital and Institute, Beijing, China; ${ }^{{ }^{D}}$ Department of Gastrointestinal Oncology, Affiliated Hospital Cancer Center, Academy of Military Medical Sciences, Beijing, China; ${ }^{\mathrm{R}}$ Department of Interventional Oncology, the First Affiliated Hospital, Sun Yat-sen University, Guangzhou, China; ${ }^{\mathrm{S} D e p a r t m e n t}$ of Hepatic Surgery \& Interventional Radiology, Eastern Hepatobiliary Surgery Hospital, Second Military Medical University, Shanghai, China; 'Department of Hepatobiliary Surgery, Zhongshan Hospital of Xiamen University, Hubing South Road, Xiamen, China; UDepartment of Surgery, Hepatic Surgery Center, Tongji Hospital, Tongji Medical College, Huazhong University of Science and Technology, Wuhan, China; ${ }^{\vee}$ Department of Hepatobiliary Surgery Institute, Southwest Hospital, Third Military Medical University, Chongqing, China; ${ }^{\text {WDepartment }}$ of Hepatobiliary and Pancreatic Surgery, the First Affiliated Hospital of Zhengzhou University, ZhengZhou, China; ${ }^{\mathrm{X}}$ Department of Hepatobiliary Surgery, Tianjin Medical University Cancer Institute and Hospital, Tianjin, China; ${ }^{Y}$ Department of Oncology, Guang'anmen Hospital, China Academy of Chinese Medical Sciences, Beijing, China; ${ }^{\mathrm{Z} D e p a r t m e n t}$ of Hepatobiliary Surgery, Peking University People's Hospital, Beijing, China; ${ }^{\alpha}$ Department of Minimally Invasive Interventional Radiology, the Second Affiliated Hospital of Guangzhou Medical University, Guangzhou, China; ${ }^{\beta}$ Department of Radiology, Zhongda Hospital, Medical School, Southeast University, Nanjing, China; YDepartment of Abdominal Surgical Oncology, Cancer Hospital, Chinese Academy of Medical Sciences and Peking Union Medical College, Beijing, China; ${ }^{\delta}$ Department of Hepatobiliary and Pancreatic Surgery, First Affiliated Hospital, School of Medicine, Zhejiang University, Hangzhou, China; ${ }^{\varepsilon}$ Department of General Surgery, Sir Run Run Shaw Hospital, Zhejiang University, Hangzhou, China; 'Department of Medical Oncology, PLA Cancer Center, Nanjing Bayi Hospital, Nanjing, China; ${ }^{\ominus}$ Department of Hepatobiliary and Pancreas Surgery, Beijing Tsinghua Changgung Hospital (BTCH), School of Clinical Medicine, Tsinghua University, Beijing, China

\title{
Keywords
}

Cancer $\cdot$ Carcinoma $\cdot$ China $\cdot$ Liver $\cdot$ Treatment $\cdot$ Diagnosis

\author{
Abstract \\ Background: Primary liver cancer, around $90 \%$ are hepatocellular carcinoma in China, is the \\ fourth most common malignancy and the second leading cause of tumor-related death, \\ thereby posing a significant threat to the life and health of the Chinese people. Summary:
}


Zhou et al.: 2019 HCC Guidelines in China

Since the publication of Guidelines for Diagnosis and Treatment of Primary Liver Cancer (2017 Edition) in 2018, additional high-quality evidence has emerged with relevance to the diagnosis, staging, and treatment of liver cancer in and outside China that requires the guidelines to be updated. The new edition (2019 Edition) was written by more than 70 experts in the field of liver cancer in China. They reflect the real-world situation in China regarding diagnosing and treating liver cancer in recent years. Key Messages: Most importantly, the new guidelines were endorsed and promulgated by the Bureau of Medical Administration of the National Health Commission of the People's Republic of China in December 2019.

(C) 2020 The Author(s).

Published by S. Karger AG, Basel

\section{Overview}

In China, primary liver cancer is the fourth most common malignancy and the second leading cause of tumor-related death, thereby posing a significant threat to the life and health of the Chinese people $[1,2]$. The main pathological subtypes of primary liver cancer are hepatocellular carcinoma (HCC), intrahepatic cholangiocarcinoma (ICC), and HCC-ICC. These 3 subtypes vary greatly in pathogenesis, biological behavior, histologic morphology, treatment methods, and prognosis. HCC is the most common primary liver cancer, accounting for $85-90 \%$ of all cases, and therefore, the remainder of this guideline refers to HCC only.

In an effort to standardize the diagnosis and treatment of HCC in China, the Bureau of Medical Administration of the former National Health and Family Planning Commission of the people's Republic of China promulgated the Guidelines for Diagnosis and Treatment of Primary Liver Cancer (2017 Edition) in June 2017. Since then, additional high-quality evidence has emerged with relevance to the diagnosis, staging and treatment of HCC in and outside China and in particular research directly applicable to clinical practice in China. In response to these developments, the Bureau of Medical Administration of the National Health Commission of the People's Republic of China entrusted the Society of Liver Cancer of China to organize a nationwide committee of multidisciplinary experts to produce Guidelines for The Diagnosis and Treatment of Hepatocellular Carcinoma (2019 Edition) (hereinafter referred to as the "Guidelines"). The levels of evidence quoted in these guidelines are based on the Oxford Centre for Evidence-Based Medicine Levels of Evidence (2009).

\section{Screening and Diagnosis}

\section{Monitoring and Screening of High-Risk Populations}

Screening for HCC in high-risk populations facilitates early detection, early diagnosis, and early treatment and is critical for improving the outcomes of patients with HCC. In China, high-risk populations mainly comprise people with hepatitis B virus (HBV) and/or hepatitis $\mathrm{C}$ virus (HCV) infection, excessive alcohol consumption, nonalcoholic fatty hepatitis, longterm consumption of aflatoxin-contaminated food, liver cirrhosis of all causes, and a family history of HCC. In particular, Chinese men $>40$ years of age are at an increased risk of developing HCC. Liver ultrasonography (US) and serum alpha-fetoprotein (AFP) testing are used for the early screening of HCC, and screening at a minimum of every 6 months is recommended in high-risk populations [3].

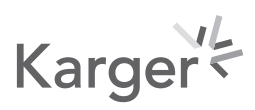


Imaging Examinations for HCC

Imaging examinations should be applied using the principle of integrated application, with different methods used to complement each other in performing an overall evaluation.

\section{Ultrasonography}

US is the most commonly used liver imaging method in clinical practice as it offers ease of operation, real-time results, noninvasiveness, and portability. A number of different US techniques exist with different clinical utility. Routine gray-scale US can detect early-stage focal liver lesions with a high degree of sensitivity and can accurately identify lesions as cystic or parenchymal, benign, or malignant. Gray-scale US is also able to detect metastatic disease in the liver or abdominal cavity and identify the invasion of intrahepatic vessels and bile ducts. Color Doppler flow US imaging can be used to visualize the blood supply within a lesion, and furthermore can identify the nature of a lesion and its adjacent relationship with important intrahepatic blood vessels. Contrast-enhanced US can visualize the hemodynamic changes within a liver tumor and provide valuable information for making differential diagnoses. In addition, contrast-enhanced US has utility for evaluating microvascular perfusion of liver tumors as well as guiding interventional therapy and evaluating treatment effect. Combining US with MRI through advanced volume navigation software provides an effective means for accurate localization of liver tumors and real-time minimally invasive ablation of HCC [4] (level 3 evidence). Intraoperative US and intraoperative contrast-enhanced US have enough sensitivity to detect micro-tumors (around $5 \mathrm{~mm}$ in diameter) in the liver and allow coordination during surgical treatment [5] (level 3 evidence). Ultrasound elastography allows noninvasive measurement of the stiffness of liver parenchyma and focal lesions and provides additional information when determining the feasibility of surgical treatment [6, 7] (level 3 evidence). Integration of multiple US techniques plays an important role in accurate preoperative diagnosis, intraoperative localization, and postoperative evaluation of HCC [8].

\section{Computed Tomography and MRI}

Dynamic contrast-enhanced computed tomography (CT) and multimodal MRI scans are the first-choice imaging methods for diagnosing patients with abnormal liver US and serum AFP screening results. In addition to wide application in the clinical diagnosis and staging of HCC, dynamic contrast-enhanced CT is also used to evaluate the response of HCC to locoregional treatment, in particular in the observation of deposition of iodized oil following transarterial chemoembolization (TACE). Furthermore, CT has other common uses in clinical practice such as 3D vascular reconstruction, measurement of liver volume, and tumor volume, and evaluation of extrahepatic metastasis.

Multimodal MRI is the preferred imaging technique for the detection, diagnosis, staging, and response evaluation of HCC as it does not involve ionizing radiation and has a high tissue resolution. In addition, MRI is multidirectional and allows sophisticated techniques such as multiparameter imaging that combines morphological images with functional imaging such as diffusion weighted imaging. Multimodal MRI has a better ability to detect and diagnose smaller liver tumors (diameter $\leq 2.0 \mathrm{~cm}$ ) than dynamic contrast-enhanced CT $[9,10]$ (level 3 evidence).

The detection rate for liver tumors $\leq 1.0 \mathrm{~cm}$ diameter and the accuracy of diagnosis and differential diagnosis of HCC are improved by using the hepatocyte-specific MRI contrast agent gadoxetic acid disodium (Gd-EOB-DTPA) [11-15] (level 2 evidence). Compared with dynamic contrast-enhanced CT, multimodal MRI is superior for evaluating involvement of the portal vein, and main trunk and branches of the hepatic vein, as well as identifying abdominal or retroperitoneal lymph node metastases.

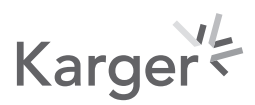


The characteristic "wash in and wash out" enhancement pattern is a key criteria for making a diagnosis of HCC from imaging data [16-18] (level 1 evidence). On dynamic contrastenhanced CT and MRI images, liver tumors exhibit a distinct homogeneous or inhomogeneous "wash in" enhancement in the arterial phase (mainly in the late arterial phase) and a "wash out" in the portal venous phase and/or equilibrium phase. Gd-EOB-DTPA-enhanced MRI exhibits enhancement in liver tumor(s) in the arterial phase, washout in the portal venous phase and, frequently, obvious hypointensity in the hepatobiliary phase with only $5-12 \%$ of small well-differentiated HCCs showing hyperintensity associated with contrast agent uptake in the hepatobiliary phase [19] (level 3 evidence).

Diagnosis of HCC by contrast-enhanced MRI requires confirmation against other characteristic imaging findings such as capsule-like enhancement, moderate hyperintensity on T2-weighted imaging, and diffusion restriction [20] (level 3 evidence). Hypointensity in the hepatobiliary phase and enhancement in the arterial phase of Gd-EOB-DTPA-enhanced MRI and diffusion restriction significantly improves the diagnostic sensitivity for small liver tumors and helps differentiate precancerous lesions such as highly dysplastic nodules [21] (level 3 evidence). Approximately $5-12 \%$ of HCCs can be found with iso- or hyperintense owing to the overexpression of OATP1B3 during hepatocarcinogenesis. Hepatobiliary iso- or hyperintense HCC was reported to be related to the activation of $\beta$-catenin or Wnt/ $\beta$-catenin target genes, consequently indicating a moderate differentiated grade and better outcome $[22,23]$. The establishment of radiomic nomograms based on mining of CT and/or MRI data from cases of HCC may help improve clinical decision-making such as selection of treatment regimens, efficacy evaluation, and prognosis [24-26] (level 3 evidence).

Digital Subtraction Angiography

Digital subtraction angiography (DSA) is an invasive procedure and in most cases is recommended to be performed through selective or ultraselective cannulation of the hepatic artery. This technique is most commonly used for delivering hepatic locoregional therapy or for the treatment of acute bleeding from tumor rupture. DSA not only visualizes liver tumor blood vessels and liver tumor staining but also allows visualization of the number, size and blood supply of liver tumors. DSA provides accurate and objective information on vascular anatomic variation and the anatomic relationship between liver tumor(s) and important blood vessels. It can also be used to assess infiltration of the portal vein, which is an important factor for judging the feasibility and prognosis for surgical resection and determining a suitable treatment regimen.

Nuclear Medicine Imaging

1. Positron emission tomography/computed tomogrpahy (PET/CT): The advantages of whole-body 18F-fluorodeoxyglucose (18F-FDG) PET/CT lie in the following: (1) Tumor staging - one procedure enables the overall evaluation of the presence of lymph node metastasis and distal organ metastasis [27, 28] (level 1 evidence). (2) Re-staging - the PET/CT functional image can accurately visualize tumor recurrence or metastases that occur following the changes of anatomic structures or at sites with a complicated anatomic structure since this imaging technique is not affected by anatomic structures $[29,30]$ (level 2 evidence). (3) Response evaluation - compared with other imaging modalities, PET/CT is more sensitive and accurate for the evaluation of response to medical interventions such as targeted drugs that act to inhibit tumor activity [31,32] (level 2 evidence). (4) Guiding biological target volume delineation for radiation therapy and determination of puncture biopsy sites [29, 30] (level 2 evidence). (5) Evaluation of the extent of malignancy and prognosis [33-36] (level 2 evidence). Carbon-11 acetate or choline PET provides improved sensitivity for the diagnosis of well-differentiated HCC and is complementary to ${ }^{18}$ F-FDG PET/CT $[37,38]$. 
2. Single-photon emission computed tomography/computed tomography (SPECT-CT): SPECT/CT has gradually become a mainstream device for nuclear medicine single-photon imaging in place of SPECT when bone metastasis was suspected in patient with liver carcinoma. The bone lesions detected by whole-body bone planar imaging can be selected for regional SPECT/CT fusion imaging, which significantly improves the accuracy of diagnosis by simultaneously obtaining the SPECT and diagnostic CT images of the lesion site [39] (level 3 evidence).

3. Positron emission tomography/MRI (PET/MRI): PET/MRI imaging provides both anatomical and functional information about the disease and improves the diagnostic sensitivity for HCC [40].

\section{Liver Biopsy}

Diagnostic liver biopsy is usually not necessary in patients with space-occupying lesions that have typical imaging characteristics and are evaluable using the clinical criteria for the diagnosis of HCC $[41,42]$ (level 1 evidence). For patients with resectable HCC or scheduled for liver transplantation, preoperative liver biopsy is not recommended, in order to reduce the risk of tumor dissemination. For space-occupying lesions without typical imaging characteristics, liver biopsy can provide a definitive pathologic diagnosis. Liver biopsy also provides valuable information on the nature of the lesion and etiology of disease, allows molecular classification of HCC [43], and can provide guidance for treatment selection and prognosis.

Liver biopsy should be performed under the guidance of US or CT with an 18G or 16G needle. The major risks of liver biopsy are bleeding and needle tract implantation. Platelet count and blood clotting should be assessed preoperatively and liver biopsy is contraindicated in patients with hemorrhagic tendency. In order to avoid tumor rupture and needle tract implantation, coaxial needles can be selected to guide the puncture, followed by embolization of the needle tract with a gelatin sponge, and normal liver tissues should be passed in the selection of puncture tract to avoid direct puncture of the nodules located on the surface of the liver. Tissue specimens from both the tumor tissue and the adjacent liver tissue should be obtained to improve the accuracy of pathological diagnosis. In addition, pathologic diagnosis by liver lesion biopsy is associated with a certain false-negative rate due to multiple factors including the size of lesion; lesions with a diameter of $\leq 2 \mathrm{~cm}$ have a high false-negative rate. Therefore, a negative result from liver biopsy cannot exclude the possibility of HCC, and follow-ups need to be conducted with an interval of 3 months. Repeat liver biopsy is recommended in patients with limited biopsy specimens and negative pathological result, but who are clinically highly suspected of having HCC.

\section{Serum Biomarkers for HCC}

Currently, serum AFP is a commonly used and important biomarker for the diagnosis of $\mathrm{HCC}$ and monitoring of treatment response. A serum AFP level of $\geq 400 \mu \mathrm{g} / \mathrm{L}$ is highly suggestive of HCC after excluding pregnancy, chronic or active liver diseases, embryonal tumors of the gonads, and gastrointestinal tumors. Patients with mildly increased serum AFP should be actively monitored. Cross comparison with changes in liver function should also be performed to facilitate diagnosis. Lens culinaris agglutinin-reactive fraction of AFP (AFP-L3), protein induced by vitamin $\mathrm{K}$ absence/antagonist-II (PIVKA II, also called des-gamma carboxyprothrombin) and plasma-free microRNA [44] may also act as diagnostic biomarkers during the early stages of HCC, in particular the serum AFP negative population. In recent years, "liquid biopsy" (circulating cell-free microRNA, circulating tumor cells, and circulating tumor DNA, etc.) has shown value in the early diagnosis and response evaluation of HCC [45].

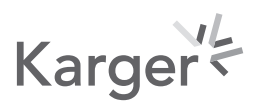


Summary of Main Points

1. US in combination with serum AFP testing is used for the early screening of HCC. Monitoring at a minimum frequency of every 6 months is recommended in high-risk populations.

2. Dynamic contrast-enhanced CT and multimodal MRI scans are the first-choice imaging methods for the diagnosis of HCC in patients with abnormal liver US and serum AFP screening results.

3. The characteristic "rapid in and rapid out" enhancement pattern is the main basis for an imaging diagnosis of HCC.

4. Multimodal MRI is the preferred imaging technique for detection, diagnosis, staging, and response evaluation of HCC.

5. PET/CT facilitates HCC staging and evaluation of response to medical interventions.

6. Liver biopsy for diagnostic purposes is usually not necessary in patients with space-occupying lesions with typical imaging characteristics and eligible for a clinical diagnosis of HCC.

7. In the serum AFP negative population, AFP-L3, PIVKA II, and plasma free microRNA can be helpful to establish an early diagnosis of HCC.

\section{Pathologic Diagnosis of HCC}

Tissue specimens obtained from biopsy or surgical resection are diagnosed as HCC through histopathologic and/or cytologic examination. The requisition form for pathologic examination should describe the patient's history of HBV/HCV infection, serum biomarkers for HCC and imaging examinations. The guidelines for pathologic diagnosis of HCC are composed of specimen handling, specimen sampling, histologic examination, and pathology report.

Specimen Handling and Specimen Sampling for HCC

1. The main guidance for specimen handling includes the following. (1) The surgeon should indicate the site, type, and number of submitted specimens on the pathology requisition form. The surgical margin and important lesions can be stained with dyes or labeled with sutures. (2) Where possible, the intact tumor specimens should be delivered to the pathologist for dissection and fixation within $30 \mathrm{~min}$ after removal. (3) Tissue samples should be fixed in 10\% neutral formalin solution for 12-24 h. (4) Liver puncture biopsy tissue samples should be placed on a piece of paper before fixation in fixative solution to prevent shrinking or bending fractures.

2. The main guidance for specimen sampling is the following. The area adjacent to HCCs is the representative area for biological features of tumor [46]. To this end, the "7-point" sampling method (Fig. 1) should be employed, that is, specimens are collected in a ratio of $1: 1$ in the $12,3,6$, and 9 o'clock positions along the boundary between cancerous and adjacent non-neoplastic liver tissues. At least 1 tissue sample should be collected from inside the tumor. One sample should also be collected from the liver tissues in the nonneoplastic adjacent regions both $\leq 1 \mathrm{~cm}$ (proximal) and $>1 \mathrm{~cm}$ (distal) from the tumor boundary. For solitary tumors with a diameter $\leq 3 \mathrm{~cm}$, the whole tumor should be sampled. In addition, the actual site and number of specimens obtained must consider the diameter and total number of tumors [47] (level 2 evidence). The "7-point" sampling method and MVI pathologic grading diagnosis are not applicable to HCCs with limited non-neoplastic adjacent liver tissues. 
Fig. 1. Schematic diagram of the recommended baseline specimen sampling protocol for liver tumors. A, B, C, and D denote the 12, 3,6 , and 9 o'clock positions, respectively, along the boundary between cancer and adjacent nonneoplastic liver tissue; E: tumor area; F: proximal non-neoplastic adjacent liver tissue; G: distal non-neoplastic adjacent liver tissue.

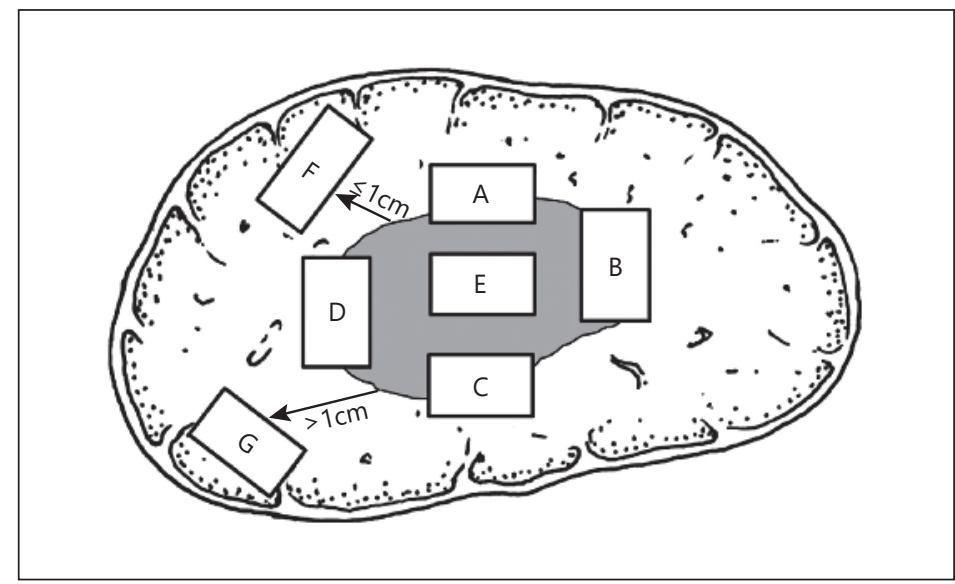

Recommendations for the Pathologic Diagnosis of HCC

1. Macroscopic description of specimens [48]: the following details should be specifically described: size, number, color and texture of tumors, their relationship with blood vessels and bile ducts, encapsulation status, non-neoplastic liver tissue lesions, type of liver cirrhosis, distance between tumor and incisal margin, and involvement of the incisal margin.

2. Microscopic diagnosis (based on the 2019 WHO diagnostic criteria for HCC): the following information should be specifically described. (1) The degree of differentiation of tumor cells can be described according to the 2019 WHO classification [49] or EdmondsonSteiner grading. (2) The histological morphology of HCC is usually divided into microtrabecular, macrotrabecular, pseudoglandular, and compact types. (3) Special types of HCC include fatty change, clear cell, macrotrabecular-massive, cirrhotic, chromophobe cell, fibrolamellar, neutrophil-rich, and lymphocyte-rich types. (4) Degree and range of tumor necrosis, lymphocyte infiltration, and stromal fibrosis. (5) The growth pattern of HCC including perineoplastic infiltration, capsule invasion or breakthrough, MVI, and presence of satellite nodules. (6) Evaluation of chronic liver disease in adjacent liver tissues (HCC is often accompanied by varying degrees of chronic viral hepatitis or liver cirrhosis). Use of the Scheuer scoring system or the Chinese Criteria for Histologic Grading and Staging of Chronic Viral Hepatitis is recommended [50-52]. MVI refers to the presence of clusters of cancer cells in blood vessels with endothelial cell linings, commonly most pronounced in the branches of the perineoplastic portal vein (including the intra-capsular blood vessels) under microscope [53] (level 1 evidence). The extent of MVI can be graded as M0: no MVI detected; M1 (low risk group): $\leq 5$ MVIs which occur in proximal nonneoplastic adjacent liver tissues; M2 (high-risk group): >5 MVIs in proximal or distal nonneoplastic adjacent liver tissues. Satellite lesions in non-neoplastic adjacent liver tissues should be included in the MVI grading in cases where it is difficult to distinguish satellite lesions from MVI [54]. MVI has a great impact on the evaluation of recurrence risk and on the selection of appropriate treatment strategy and should be considered a routine parameter for pathologic examination [55-57] (level 2 evidence).

3. Immunohistochemical examination: immunohistochemical marker panels should be used in a proper combination for differential diagnosis of HCC, ICC, HCC-ICC, and metastatic HCC [48]. The recommended hepatocyte markers include arginase-1 (Arg-1), hepatocyte paraffin 1 (Hep Par1), glypican-3 (GPC-3), AFP, polyclonal CEA (pCEA) and CD10. The most commonly used markers for early-stage HCC include GPC-3, HSP70, and glutamine synthetase (GS). Immunohistochemical staining for programmed death-1 (PD-1) and programmed death ligand-1 (PD-L1) are also recommended [58]. 
4. Molecular testing: Molecular targets allowing individualized selection of targeted drugs are currently under preclinical development and validation. For example, the pattern of clonal origin of multinodular HCC and postoperative recurrent HCC influences clinical staging and selection of treatment regimens $[59,60]$. However, it is difficult to identify the clonal origin by conventional histomorphological observation. For this reason, genome loss of heterozygosity of microsatellites can be used to evaluate the clonal origin of multinodular HCC and postoperative recurrent HCC and provide reference for clinical staging and selection of individualized treatment regimens $[61,62]$.

Pathologic Diagnosis Report

A typical pathological report should include gross description of specimens, microscopic description, immunohistochemical staining examination results, and the final pathologic diagnosis. In addition, molecular examination results related to the clonal origin of HCC, drug target testing, biological behavior evaluation, and prognosis assessments can be attached for clinical reference.

Summary of Main Points

1. Standardized handling and timely delivery of biopsy/resected tissue samples are of great significance for tissue preservation and correct diagnosis.

2. The "7-point" sampling method should be followed.

3. The contents of pathologic diagnosis reports for HCC should be standardized and comprehensive and specifically include the pathological classification of MVI.

\section{Criteria for Clinical Diagnosis of HCC and Diagnostic Pathway}

A clinical diagnosis of HCC should be established in accordance with the steps shown in the following pathway, taking into account the high-risk factors for HCC, imaging characteristics, and serological molecular markers (Fig. 2).

1. Screening through US and serum AFP testing should be performed at a minimum of every 6 months in patients with viral HBV/HCV or liver cirrhosis of all causes. For patients with nodules of a diameter of $\leq 2 \mathrm{~cm}$, a clinical diagnosis of HCC can be established by observation of the "rapid in and rapid out" enhancement pattern on contrast-enhanced imaging (enhancement in the arterial phase and reduced enhancement of intrahepatic lesions compared with healthy liver parenchyma in the portal venous and/or balance phase). This pattern should be observed on at least 2 of the following 4 imaging examinations; dynamic contrast-enhanced MRI, CT, and US and contrast-enhanced MRI using the hepatocyte-specific contrast agent Gd-EOB-DTPA. For intrahepatic nodules with a diameter of $>2 \mathrm{~cm}$, a clinical diagnosis of HCC can be established when the "rapid in and rapid out" enhancement pattern is observed on any of these 4 imaging examinations.

2. For patients with HBV/HCV or liver cirrhosis of all causes and intra-hepatic nodules with a diameter $\leq 2 \mathrm{~cm}$ observed during follow-up, a diagnosis can be established by liver puncture biopsy or 2- to 3-monthly imaging examinations in combination with serum AFP testing if the typical enhancement characteristics of HCC are noted in zero or one of the 4 imaging examinations. For patients with intrahepatic nodules with a diameter of $>2$ $\mathrm{cm}$, a diagnosis can be made by liver lesion puncture biopsy if the typical enhancement characteristics of HCC are not observed in any of the 4 imaging examinations.

3. For patients with HBV/HCV, or liver cirrhosis of all causes and increased serum AFP, in particular continuously increased AFP, imaging examinations should be performed. The close monitoring of serum AFP levels and 2- to 3-monthly imaging examinations should be performed after the exclusion of pregnancy, chronic or active liver disease, embryonic reproductive tumors, and gastrointestinal cancer, if no intrahepatic nodules are identified.

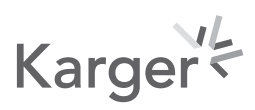


Zhou et al.: 2019 HCC Guidelines in China

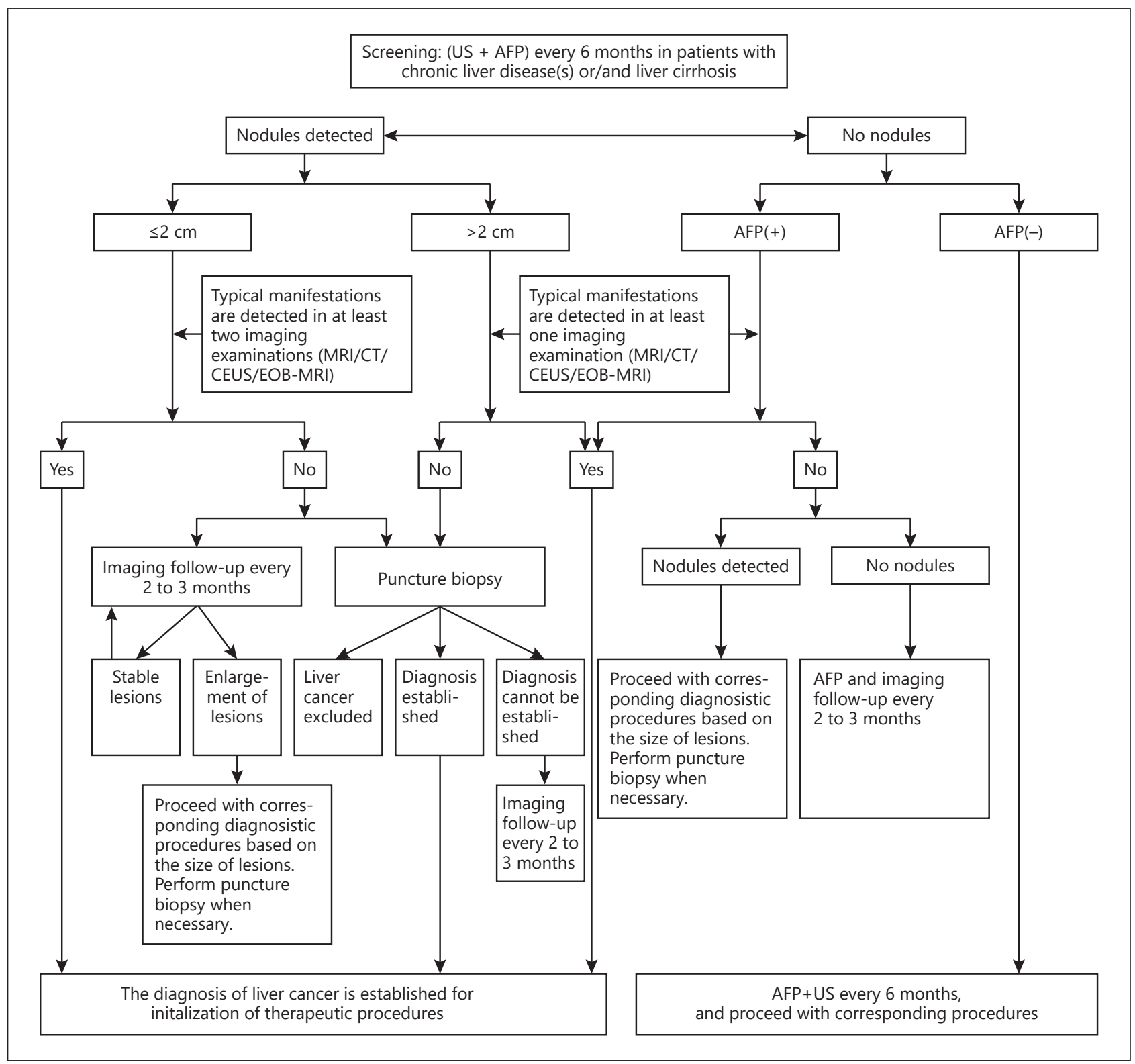

Fig. 2. Pathway for the diagnosis of HCC. Typical characteristics: the "rapid in and rapid out" enhancement pattern that manifests as enhancement of lesions in the arterial phase and reduced enhancement in the portal venous or equilibrium phase of dynamic contrast-enhanced imaging. Atypical characteristics: absence of obvious enhancement of lesions in the arterial phase of dynamic contrast-enhanced imaging, or no reduction/no obvious reduction/mild increase in enhancement in the portal venous or equilibrium phase of dynamic contrast-enhanced imaging. MRI, dynamic contrast-enhanced MRI; CT, dynamic contrast-enhanced CT; CEUS, contrast-enhanced US. Ultrasound contrast agents are used for real-time observation of blood perfusion of normal and pathological tissues. EOB-MRI, contrast-enhanced MRI with the hepatocyte-specific contrast agent gadoxetic acid disodium (Gd-EOB-DTPA). AFP(+), serum AFP above the ULN; ULN, upper limit of normal; US, ultrasonography. 
Zhou et al.: 2019 HCC Guidelines in China

\section{Staging}

The staging of HCC is crucial for prognostic assessment and selection of appropriate treatment strategy. There are a number of staging systems, including the Barcelona Clinic Liver Cancer (BCLC), TNM, Japan Society of Heptatology (JSH) and Asia Pacific Association for the Study of the Liver (APASL) staging systems. The China liver cancer (CNLC) staging system takes into account the patient's general health status, and the status of liver tumors and liver function. The CNLC staging system is divided into Stage Ia, Ib, IIa, IIb, IIIa, IIIb, and IV. See Figure 3 for details.

CNLC Stage Ia: a performance status (PS) score of 0 to 2, Child-Pugh A/B liver function, a solitary tumor with a diameter of $\leq 5 \mathrm{~cm}$, and absence of vascular invasion or extrahepatic metastasis.

CNLC Stage Ib: a PS score of 0 to 2, Child-Pugh A/B liver function, a solitary tumor with a diameter of $>5 \mathrm{~cm}$, or 2 to 3 tumors with a maximum diameter $\leq 3 \mathrm{~cm}$, and absence of vascular invasion or extrahepatic metastasis.

CNLC Stage IIa: a PS score of 0 to 2, Child-Pugh A/B liver function, 2 to 3 tumors with a maximum diameter $>3 \mathrm{~cm}$, and absence of vascular invasion or extrahepatic metastasis.

CNLC Stage IIb: a PS score of 0 to 2, Child-Pugh A/B liver function, $\geq 4$ tumors regardless of tumor diameter, and absence of vascular invasion or extrahepatic metastasis.

CNLC Stage IIIa: a PS score of 0 to 2, Child-Pugh A/B liver function, regardless of tumor status, and presence of vascular invasion but absence of extrahepatic metastasis.

CNLC Stage IIIb: a PS score of 0 to 2, Child-Pugh A/B liver function, regardless of tumor status and vascular invasion, and presence of extrahepatic metastasis.

CNLC Stage IV: a PS score of 3 to 4, Child-Pugh C liver function, regardless of tumor status, vascular invasion, and extrahepatic metastasis.

\section{Treatment}

The treatment of HCC involves multiple methods and disciplines and must be led by a multidisciplinary diagnosis and treatment team, particularly for complicated cases. The selection of appropriate treatment approaches should be based on high-level evidence, while also taking into account other considerations such as regional/local economic situations and access to care.

\section{Surgical Treatment}

Surgical treatment provides the best opportunity for achieving long-term survival in HCC patients and is mainly comprised of hepatectomy and liver transplantation.

\section{Basic Principles for Hepatectomy}

1. Thoroughness: complete removal of tumor tissue, ensuring that the surgical margin is free of residual tumor.

2. Safety: preservation of a sufficient volume of functional liver tissue (with good blood supply and blood and bile outflow) to compensate liver function and reduce surgical complications and postoperative mortality.

Preoperative Evaluation of Patients' General Condition and Liver Function Reserve

Preoperative evaluation of patients' general condition and liver function reserve (LFR) is mandatory. Eastern Cooperative Oncology Group PS (ECOG PS) is commonly used to evaluate the patient's general condition. Liver function evaluation, such as Child-Pugh Score, indocy-

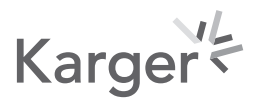


General

health status

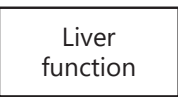

Extrahepatic

metastases

Blood vessel invasion
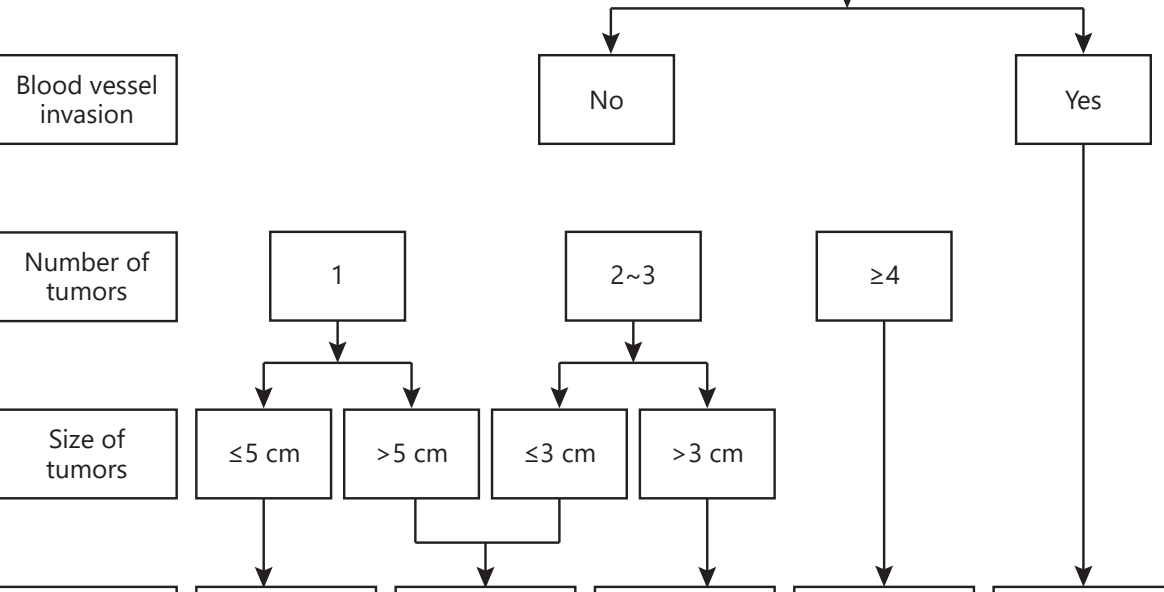

Size of
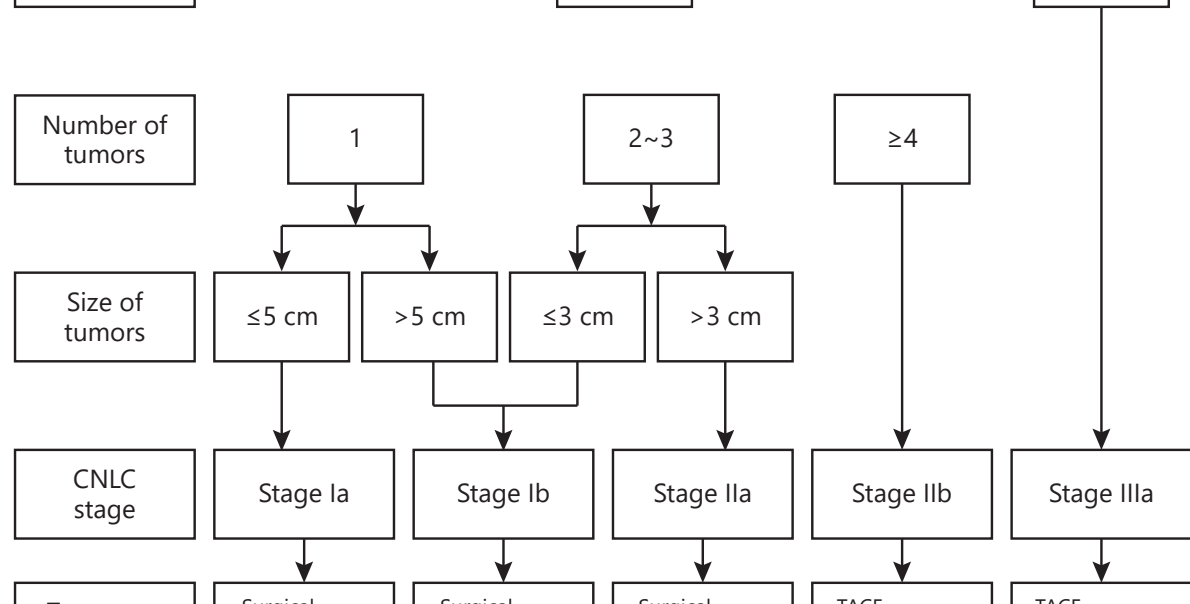

Treatment
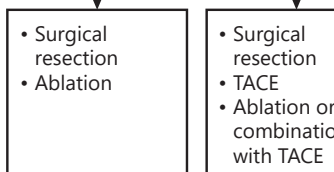

- TACE

- Ablation or in

combination

with TACE
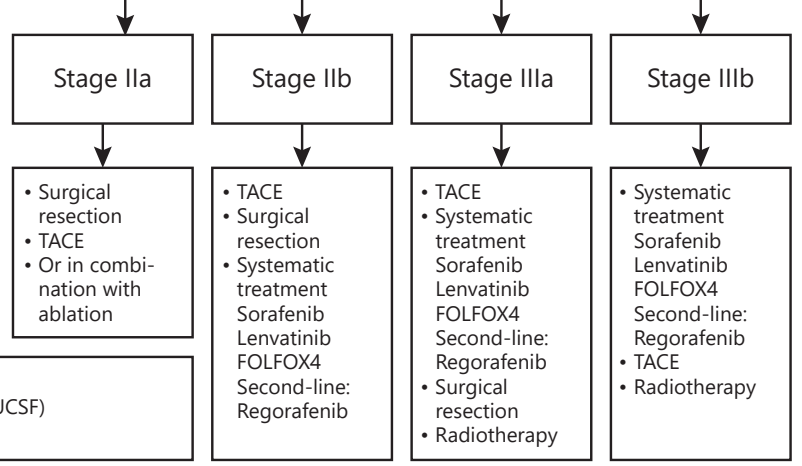

CC
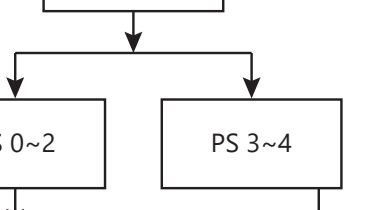

- Liver transplantation (UCSF)

Fig. 3. China clinical staging and treatment pathway for HCC. Notes: FOLFOX4, 5-fluorouracil, leucovorin, and oxaliplatin; HCC, hepatocellular carcinoma; PS, performance status; TACE, transarterial chemoembolization; UCSF, University of California, San Francisco.

anine green (ICG) clearance test, or transient elastography for liver stiffness measurement [63-68] is used to evaluate LFR. Many studies, including those from Chinese researchers, suggest that selected patients with portal hypertension can still receive hepatectomy, and that their long-term survival following surgery is superior to that associated with other treatments $[69,70]$. Therefore, an accurate evaluation of the degree of portal hypertension [71, 
Zhou et al.: 2019 HCC Guidelines in China

72] is helpful to screen patients suitable for surgical resection. CT and/or MRI is used to measure the remaining liver volume and for calculation of the percentage of the remaining liver volume versus the standardized liver volume if the preservation of a small volume of liver tissues is expected. Child-Pugh Grade A and ICG-R15 $<30 \%$ are generally believed to be the prerequisites for successful surgical resection. The remaining liver volume that accounts for $>40 \%$ (for patients with liver cirrhosis) or $>30 \%$ (for patients without liver cirrhosis) of the standardized liver volume is another prerequisite for surgical resection.

\section{Indications for Hepatic Carcinectomy}

1. CNLC Stage Ia, Ib, and IIa HCC and good LFR are the most important indications for successful surgical resection. The results from recent studies have shown surgical resection is associated with a significantly lower local recurrence rate than radiofrequency ablation and suggest that the lack of difference in long-term survival between both treatments may be attributable to the use of additional curative treatments following recurrence [73] (level 2 evidence). However, older studies indicate equivalent efficacy for surgical resection versus local ablation for HCCs with a diameter $\leq 3 \mathrm{~cm}$ [74] (level 1 evidence). In addition, multiple observational studies have shown that surgical resection is associated with better long-term outcomes [75-77] (level 1 evidence).

2. In most cases, surgical resection is not superior to non-surgical approaches such as TACE in patients with CNLC Stage IIb HCC. However, when tumors are localized to the same liver segment or ipsilateral hemi-liver and radiofrequency ablation can be performed to preserve more functional liver volume, surgical removal may be superior to other treatment approaches even with multiple tumors $(>3)[78,79]$.Therefore, in this case, surgical removal is also recommended (level 2 evidence). However, a very thorough preoperative evaluation is recommended in these cases.

3. For CNLC Stage IIIa HCCs, surgical removal can be considered in the following circumstances. (1) In patients with tumor thrombi in the main trunk or branches of the portal vein, surgical removal of the tumor and embolectomy through the portal vein can be considered, followed by postoperative TACE, portal vein chemotherapy, or other systemic treatments when the tumor is localized to the hemi-liver with tumor thrombi formed in the branches of the portal vein (Cheng's Classification type I/II) [80]. Surgical resection of tumor thrombi in the main portal vein (Cheng's Classification type III) remains controversial and may produce an outcome equivalent to TACE or external radiotherapy. Therefore, the presence of tumor thrombi in the trunk of the portal vein is not an absolute indication for surgical resection [81] (level 3 evidence). A randomized controlled study has shown that preoperative three-dimensional conformal radiotherapy is associated with improved postoperative survival in resectable patients with portal vein tumor thrombus [82] (level 2 evidence). (2) Patients with tumor thrombi in the bile duct and obstructive jaundice and resectable intrahepatic lesions. (3) For patients with portal lymph node metastases, intraoperative lymph node dissection or postoperative external radiation therapy can be performed in addition to tumor resection. (4) Patients with involvement of adjacent organs that can be resected simultaneously.

In addition, hepatic artery and portal vein catheterization chemotherapy or other intraoperative locoregional treatments can be considered for HCC that is confirmed as unsuitable for resection during surgical exploration.

Criteria for Curative Resection

1. Intraoperative assessment criteria: (1) no macroscopic tumor thrombi are noted in the hepatic vein, portal vein, bile duct, and inferior vena cava. (2) In the absence of adjacent organ involvement, portal lymph node or distal metastases. (3) The distance between the 
surgical margin and tumor boundary is $>1 \mathrm{~cm}$ or histologic examination of the cross section of the resected liver is free of residual tumor cells, that is, negative surgical margin, if the surgical margin is $<1 \mathrm{~cm}$.

2. Postoperative judgment criteria: (1) US, CT, and MRI (at least 2 of these 3 examinations are mandatory) performed 2 months after surgery confirm the absence of tumor lesions. (2) Quantitative AFP testing should be performed postoperatively at 2 months to ensure AFP is within normal range (it should be noted that the time to normalization of AFP is $>2$ months in isolated patients) if the AFP levels were elevated preoperatively. The rate of decrease in serum AFP can predict the thoroughness of surgical resection [83].

Surgical Resection Techniques

Commonly used hepatectomy techniques include hepatic inflow and outflow control techniques, liver transection techniques and hemostatic techniques. Preoperative threedimensional visualization technology helps to design a more accurate path of resection enabling protection of the ducts of the remaining liver and achieving a better oncological outcome (level 3 evidence). Laparoscopic hepatectomy has advantages including reduced invasiveness and more rapid recovery [84] (level 2 evidence). A retrospective study has shown that laparoscopic hepatectomy is associated with similar long-term outcomes as open surgery [85] (level 3 evidence). However, prospective, multicenter, randomized controlled studies are required to support this approach. There is evidence supporting improved prognosis following laparoscopic hepatectomy compared with radiofrequency ablation, especially in patients with tumors located in peripheral sites. Laparoscopic hepatectomy is associated with less bleeding when conducted in experienced centers and ICG fluorescence, 3D laparoscopy, and robot assistance will become important tools for laparoscopic hepatectomy in the future, improving surgical outcomes for patients with HCC [86].

Both anatomic and nonanatomic resections are commonly used surgical techniques. Studies show that hepatectomy with wide surgical margins is associated with better outcomes than hepatectomy with narrow surgical margins (level 2 evidence) [87], especially in patients with MVI [88]. For large liver tumors, anterior approach hepatectomy without dissecting perihepatic ligaments can be performed [89]. For multiple liver tumors, surgical removal in combination with intraoperative locoregional ablation (e.g., radiofrequency ablation) can be performed [90] (level 3 evidence). For patients with portal vein tumor thrombus, the portal venous flow of the unaffected side should be temporarily interrupted during portal vein embolectomy to avoid the dissemination of tumor thrombi [91]. For patients with tumor thrombi in the hepatic vein or vena cava, total blood vessel control can be interrupted to maximize the removal of tumor thrombus [92]. For patients with tumor thrombi in the right atrium, embolectomy through the opening of the chest and right atrium can be performed along with hepatic tumorectomy. For HCC patients with tumor thrombi in the bile duct, resection of the involved bile ducts and bile duct reconstruction should be performed in addition to embolectomy to reduce the risk of local recurrence, particularly if the bile duct wall is partially invaded by the tumor [93].

Insufficient residual liver function as a result of insufficient remnant liver volume is the main factor that hinders the application of radical resection. The following methods can be used for improving the resectablility of HCC.

1. Surgical resection can be performed after induction of tumor shrinkage by preoperative TACE in some patients with Stage I HCC unsuitable for surgical resection [94, 95].

2. Portal vein embolization (PVE) should be applied to the hemi-liver in which the main tumor is located for compensatory hypertrophy of the remaining liver before resection [96]. Complications from this procedure are rarely reported in clinical practice; however, the combined use of TACE can be considered to reduce the risk of tumor progression

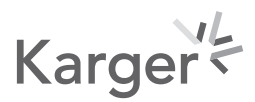


during the waiting time since 4-6 weeks are needed to allow volume increase of the contralateral liver [97].

3. Associating Liver Partition and Portal Vein Ligation for Staged Hepatectomy (ALPPS) [98] (level 3 evidence) is suitable for patients in whom remnant liver volume is $<30-40 \%$ of the standardized liver volume. Preoperative evaluation is very important and should incorporate the degree of liver cirrhosis, the patient's age, and ability to tolerate 2 surgeries within a short period of time [99]. In addition, laparoscopy or ablation techniques can be used to reduce the trauma associated with the second surgery $[100,101]$. The use of ALPPS is associated with increased incidence of complications and mortality, although it can improve the resection rate for HCC within a short period of time. Preliminary results from observational studies show that ALPPS produces better outcomes than TACE for the treatment of large or multinodular HCC. These potential benefits must be balanced against the trauma caused by receiving 2 surgeries within a short period of time, and the possibility of failure of the second-stage surgery should be considered. Extra care should be taken in the selection of appropriate candidates for this procedure.

4. For HCCs in patients with serious liver cirrhosis, those with multiple nodules, and with tumors deep in position during abdominal exploration, intraoperative locoregional ablation can reduce surgical risks.

Preoperative Treatment

For unresectable HCC, application of locoregional treatments such as TACE or external radiation therapy may result in tumor downstaging and consequently provide initially inelligible patients with opportunities for surgical intervention. Good long-term survival may be achieved in HCC patients undergoing resection after downstaging [94]. For patients with resectable HCC, preoperative TACE is not associated with improved survival $[102,103]$ (level 2 evidence).

For patients with HBV-related HCC and preoperative elevated HBV-DNA level and ALT level $>2$ times the ULN, antiviral treatments can be administered followed by surgical resection when liver function is improved to ensure safety of surgery. For patients with increased HBV-DNA copy numbers, but without obvious abnormalities in liver function, surgery can be performed as soon as possible while administering effective antiviral therapy. Anti-HBV treatment not only controls underlying liver diseases, but also reduces postoperative recurrence rates [104, 105] (level 1 evidence).

Postoperative Treatment (for Prevention and Treatment of Postoperative Metastasis and Recurrence)

The 5-year recurrence rate after surgical resection of HCC is around 40-70\%, and recurrence is often associated with preexisting minimal disseminated lesions or multicentric origin. Therefore, close follow-ups are mandatory in all patients. In the case of recurrence, repeated resection, local ablation, TACE, radiation therapy, or systemic treatment can be performed to prolong survival based on the characteristics of the recurrent disease. For patients at high recurrence risk, 2 randomized controlled studies have shown that postoperative TACE can reduce recurrence rates and prolong survival $[106,107]$ (level 2 evidence). A further randomized controlled study has shown that treatment with Huaier granules after hepatectomy is associated with reduced recurrence rates and prolonged survival [108] (level 1 evidence). Antiviral treatment with nucleoside analogs is associated with reduced recurrence and prolonged survival in HCC patients with HBV infection [104, 105, 109] (level 1 evidence). In addition, the concurrent use of portal vein catheterization chemotherapy and TACE after surgery can also prolong survival in patients with portal vein tumor thrombus [110]. The use of interferon- $\alpha$ to reduce recurrence risk and prolong survival [111-113]

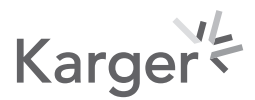


(level 2 evidence) still remains controversial [114], although such effects have been demonstrated in some randomized clinical studies. There are reports of an association between miR-26a expression in HCC and efficacy of treatment with interferon $\alpha$ [115]. However, further multicenter randomized controlled studies are warranted to confirm this result. Large clinical studies show that treatment with sorafenib fails to improve postoperative survival in patients with early stage HCC [116]; however, some small clinical studies suggest that postoperative treatment with sorafenib is associated with reduced tumor recurrence and prolonged survival in patients at high risk for recurrence [117].

Summary of Main Points

1. Hepatectomy is an important means of achieving long-term survival in patients with HCC.

2. The key principle of hepatectomy is to completely remove the tumor and preserve sufficient volumes of functional liver tissues. Thus, overall preoperative LFR evaluation and oncological evaluation are of great importance.

3. Child-Pugh Grade A and ICG-R15 < 30\% are generally accepted as prerequisites for surgical resection. A remaining liver volume accounting for $>40 \%$ (for patients with liver cirrhosis) or $>30 \%$ (for patients without liver cirrhosis) of the standardized liver volume is another prerequisite for surgical resection. Preoperative evaluation methods also include measurement of liver stiffness and degree of portal hypertension.

4. CNLC Stage Ia, Ib, and IIa HCCs in patients with good liver reserve function are the best indications for surgical resection. Surgical resection may lead to favorable outcomes in selected patients with CNLC stage IIb and stage IIIa HCC. In addition, intraoperative locoregional ablation, preoperative TACE, and preoperative conformal radiotherapy may improve resectability of CNLC Stage IIb and IIIa HCCs.

5. Hepatic inflow (hepatic artery and portal vein) and outflow (hepatic vein) control techniques are frequently used during hepatectomy. Preoperative three-dimensional visualization technology improves the accuracy of hepatectomy. Laparoscopic techniques can reduce surgical trauma; however, randomized controlled studies are still warranted to validate long-term outcomes of this approach in HCC.

6. For patients with large liver tumors and small volumes of remaining liver, preoperative TACE aiming to reduce tumor volume, portal vein embolization/ligation and ALPPS for compensatory hypertrophy of the remaining liver can be used to improve the possibility for resection.

7. For unresectable HCC locoregional therapies such as TACE and external radiation therapy can be performed to achieve tumor downstaging before surgical resection. However, preoperative TACE has not been shown to improve outcomes in patients with resectable HCC.

8. The primary goal of postoperative adjuvant therapy for HCC is to reduce recurrence. Postoperative TACE for patients at high risk for recurrence is associated with reduced recurrence and prolonged survival and postoperative oral administration of Huaier granules also reduces risk of recurrence and prolongs survival. In addition, postoperative use of nucleoside analogs for anti-HBV treatment or interferon- $\alpha$ can also reduce risk of recurrence and prolong survival.

\section{Liver Transplantation}

Indications for Liver Transplantation for HCC

Following appropriate indications is crucial for improving the outcomes of liver transplantation for HCC, ensuring fair and proper use of precious donor liver resources and balancing the presence or absence of prognostic differences between patients [118] (level 3 evidence). The Milan criteria and University of California San Francisco (UCSF) criteria are commonly used in the international community. A number of criteria have been proposed by

\section{Karger's}


Chinese experts, including the Hangzhou criteria [119], Shanghai Fudan criteria [120], WestChina criteria [121], and Sanya consensus [122]. All of these criteria agree on factors, including the absence of macrovascular involvement, lymph node metastasis, and extrahepatic metastasis, but diverge in classification by the size and number of tumors. These domestic criteria expand indications for liver transplantation for HCC to enable a greater number of HCC patients to benefit from liver transplantation without significantly reducing postoperative overall survival and tumor-free survival. However, multicenter collaborative studies are still required to reach a consensus. For now, the UCSF criteria are recommended for use and specify the diameter of a solitary tumor $\leq 6.5 \mathrm{~cm}$; the number of tumors $\leq 3$ of which the maximum tumor diameter is $\leq 4.5 \mathrm{~cm}$ and the sum of tumor diameters $\leq 8.0 \mathrm{~cm}$; no macrovascular invasion.

The development of surgical technique has led to an expansion of available donor livers. Further expansion of the indications of living donor liver transplantation for HCC can be attempted [123]; however, living donor liver transplantation for HCC may be associated with higher postoperative tumor recurrence than conventional donor surgery without obvious advantages in survival (level 4 evidence) [124].

\section{Prevention and Treatment of Post-Transplant Recurrence}

Tumor recurrence is the major concern after liver transplant for HCC [125]. Risk factors include tumor stage, vascular invasion, serum AFP level, and cumulative dose of immunodepressants. Early withdrawal of or no use of postoperative hormone-containing regimens [126] and dose reduction of calcineurin inhibitors in the early posttransplant period are associated with lower rates of tumor recurrence [127] (level 3 evidence). The use of immunosuppressive therapy with mTOR inhibitors such as rapamycin and everolimus after liver transplantation may also be associated with reduced tumor recurrence and improved survival rates [128-131] (level 3 evidence).

Upon tumor recurrence or metastasis post liver transplantation, the disease typically progresses rapidly, with a median survival of 7-16 months in patients with recurrence and metastasis [132]. The survival of patients can be prolonged by a combination of modification of immunosuppressive regimens, reoperation, TACE, local ablation, radiotherapy, and systemic treatment on the basis of multidisciplinary diagnosis and treatment [133] (level 4 evidence).

Summary of main points

1. Liver transplantation is a radical treatment approach for HCC and is particularly suitable for patients with compensated liver function.

2. It is recommended that the UCSF criteria are followed as the Chinese criteria for the indication of liver transplantation for HCC.

3. Early withdrawal of or no use of hormone-containing regimens, dose reduction of calcineurin inhibitors in the early posttransplant period and use of immunosuppressive therapy with mTOR inhibitors such as rapamycin and everolimus after liver transplantation may also be associated with reduced tumor recurrence and improved survival rates.

4. Upon tumor recurrence and metastasis post liver transplantation, the disease usually progresses rapidly. Combination treatment on the basis of multidisciplinary diagnosis and treatment is associated with prolonged survival.

\section{Local Ablation}

Although surgery provides the best survival outcomes for patients with HCC, only $20-30 \%$ of patients have opportunities for surgical resection as a large proportion have liver cirrhosis or are already at an intermediate or advanced stage at the time of diagnosis. Locore-

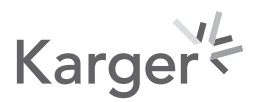


Zhou et al.: 2019 HCC Guidelines in China

gional ablation provides opportunities for radical treatment in patients with early HCC unsuitable for surgical resection.

Local ablation mainly includes radiofrequency ablation (RFA), microwave ablation (MWA), percutaneous ethanol injection (PEI), cryotherapy, high-intensity focused ultrasound ablation (HIFU), laser ablation, and irreversible electroporation (IRE). Local ablation is often performed under the guidance of US, which has advantages of ease of use, no exposure to ionizing radiation, real-time results, and high efficiency. CT, MRI, or multimodal image fusion systems can also be used for guidance, especially in cases in which lesions are invisible on conventional US. CT or MRI guidance can also be used in the ablation of metastases in the lungs, adrenal glands, and bones.

Ablation can be performed through a percutaneous, laparoscopic, or laparotomic approach. Most HCC lesions can be percutaneously ablated, which has advantages of costeffectiveness, ease of use, and minimal invasiveness. For sub-capsular HCC, in particular lesions protruding beyond the liver capsule, ablation is usually associated with a high risk of bleeding or tumor seeding. For lesions that are located at high-risk sites (close to the heart, diaphragm, gastrointestinal tract, or gallbladder) and for which protective measures such as artificial pleural effusion or ascites cannot be taken, ablation by laparoscopic or laparotomic approaches can be considered.

Local ablation is suitable for patients with CNLC Stage Ia and a proportion of those with Stage Ib HCC (i.e., solitary tumors with a diameter of $\leq 5 \mathrm{~cm}$; or 2 to 3 tumors with the maximum diameter of $\leq 3 \mathrm{~cm}$ ), no invasion of blood vessels or bile ducts, without adjacent organ invasion or distal metastasis and with Child-Pugh grade A/B liver function. Outcomes of local ablation are comparable to radical resection in these patients [74, 75, 134-137] (level 1 evidence). TACE combined with RFA can be used for patients with inoperable solitary tumors or multiple tumors with a diameter of 3-7 cm [138-140] (level 1 evidence). Postoperative adjuvant therapy with sorafenib is not recommended in patients undergoing radical ablation therapy [116] (level 1 evidence).

\section{Ablation Therapies}

1. RFA: RFA is a commonly used minimally invasive ablation method for HCC and has advantages, including ease of use, short hospital stay, excellent efficacy, and good control over the ablation range. This procedure is particularly suitable for older patients and patients with comorbid diseases, severe cirrhosis, tumors located in deep positions in the liver, or central HCC. For patients with resectable early-stage HCC, RFA is associated with similar or slightly lower tumor-free survival and overall survival but lower incidence of complications and shorter hospital stay, than surgical resection [74, 75, 134-137] (level 1 evidence). For solitary HCC with a diameter of $\leq 2 \mathrm{~cm}$, RFA has been shown to have similar or superior efficacy to surgical resection, in particular for central HCC [141, 142] (level 3 evidence). For patients with unresectable early-stage HCC, results from a systematic review and longterm studies show that RFA can lead to curative outcomes and should be recommended as the first-line treatment for early-stage HCC unsuitable for surgery [143-145] (level 1 evidence). RFA has significant advantages including a higher radical cure rate, fewer sessions of treatment, and higher long-term survival rates compared with PEI [146] (level 1 evidence). The essence of RFA is to ablate the tumor as a whole and maintain a sufficient safety margin while minimizing the damage to normal liver tissues. The range of tumor infiltration should be accurately evaluated and satellite lesions should be identified before the procedure. Therefore, the importance of accurate imaging examinations prior to treatment should be emphasized. Contrast-enhanced US can allow accurate determination of the size and shape of a tumor, define the range of tumor infiltration, and detect micro and satellite lesions, providing a reliable reference for the calculation of ablation range. 
2. MWA: MWA is another commonly used thermal ablation method, which is characterized by high efficiency, short ablation time, and reduced heat-sink effect as compared with RFA. For large tumors with rich blood supply and adjacent to large blood vessels, MWA has advantages, including short treatment time and little influence by vascular heat sinks. MWA also provides treatment opportunities for elderly patients unable to tolerate lengthy anesthesia and patients who previously received stent or pacemaker implantation. Its clinical application has gradually increased in recent years. The establishment of temperature monitoring systems helps determine the range of the effective thermal field and increase the safety of the MWA procedure. Randomized controlled studies have shown no statistically significant differences in local efficacy, rates of complications, and survival outcomes between RFA and MWA [147] (level 2 evidence). The selection of MWA or RFA should be based on the size and position of tumor, and the operator's experience [148] (level 2 evidence).

3. PEI: PEI is suitable for tumors with a diameter of $\leq 3 \mathrm{~cm}$. PEI has good ablative effects and similar long-term efficacy to RFA for tumors with a diameter of $\leq 2 \mathrm{~cm}$ despite having a higher local recurrence rate versus RFA [146] (level 1 evidence). The advantage of PEI lies in its safety and is in particular suitable for tumors in high-risk locations such as lesions near the hepatic hilar region, gallbladder, and gastrointestinal tracts. However, repeated PEI procedures are required.

Basic Technical Requirements

1. The physician must receive adequate training and have sufficient clinical experience. A thorough evaluation of the patient's general performance status and liver function, as well as evaluation of the size, position, and number of tumors should be performed prior to treatment. Attention should be paid to the relationship of the tumor with adjacent organs. Appropriate puncture tract and ablation ranges should be calculated to secure a sufficient safety margin.

2. Appropriate imaging guidance (US, CT, or multimodal image fusion) and ablation technique (RFA, MWA, or PEI) should be selected based on the size and position of the tumor and the operator's experience.

3. Ablation is suitable for tumors located at least $5 \mathrm{~mm}$ from the common hepatic duct or the left/right hepatic duct in the hepatic hilar region. For lesions with a diameter $>5 \mathrm{~cm}$, TACE in combination with ablation is recommended, which provides better outcomes than ablation therapy alone.

4. The range of ablation should cover at least $5 \mathrm{~mm}$ of perineoplastic liver tissue to ensure a safety margin and achieve a complete ablation. For ill-defined and irregularly infiltrating tumors, the range of ablation is recommended to be extended as appropriate if adjacent liver tissues and structures permit.

Treatment Recommendations for Tumors $\leq 5 \mathrm{~cm}$

Several randomized controlled trials and retrospective analyses support surgical resection as the first choice for resectable early HCC [75-77, 134, 136] (level 1 evidence). In real-world clinical practice, initial treatment should be selected after a thorough consideration of the patient's general performance status and liver function and the size, number and position of tumors, as well as the skills and experience of the physician. Surgical resection is the first choice if the patient can tolerate hepatectomy or the tumors are located in a superficial area, the peripheral liver, or high-risk sites unsuitable for ablation. Local ablation is the preferred choice for deeply located or central tumors or for patients with 2-3 tumors located in different liver segments/lobes and can have an efficacy equivalent to surgical resection with minimal invasiveness in these cases.

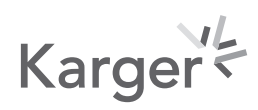


Evaluation and Follow-Up after Ablation Therapy

Dynamic contrast-enhanced CT or MRI, or contrast-enhanced US is recommended for assessing the response to ablation around 1 month after the procedure. Dynamic changes of serum AFP level should be monitored in patients with elevated baseline AFP. The response to ablation can be categorized into the following [149]. (1) Complete ablation: follow-up imaging shows no enhancement in the ablated area of the tumor in the arterial phase, which indicates complete necrosis of the tumor. (2) Incomplete ablation: follow-up imaging shows local enhancement in the ablated region of the tumor in the arterial phase, which is suggestive of residual tumor. Repeat ablation is suggested in this situation. Ablation therapy should be abandoned and switched to other treatments if the presence of residual tumor is still confirmed after 2 consecutive ablation sessions. Periodic follow-ups are required after complete ablation is achieved. Serum tumor marker evaluation and imaging examination should be performed every 2-3 months to screen for local recurrence and new intrahepatic lesions. Ablation therapy can be repeated due to its effectiveness, minimal invasion and good safety profile.

Summary of main points

1. Local ablation therapy is suitable for patients with CNLC Stage Ia and a proportion of patients with Stage Ib HCC (i.e., solitary tumors with a diameter of $\leq 5 \mathrm{~cm}$ or $2-3$ tumors with maximum diameter $\leq 3 \mathrm{~cm}$ ); no invasion of blood vessels and bile ducts; without adjacent organ invasion or distal metastasis and Child-Pugh Grade A/B liver function. Outcomes of ablation therapy are comparable to those of radical resection in these selected patients. TACE combined with ablation can be used for inoperable solitary or multiple tumors with a diameter of 3-7 cm. Postoperative adjuvant therapy with sorafenib is not recommended in patients undergoing radical ablation therapy.

2. For patients with resectable early-stage HCC, RFA is associated with similar or slightly lower tumor-free survival and overall survival, but lower incidence of complications and shorter hospital stay than surgical resection. For solitary HCC with a diameter $\leq 2 \mathrm{~cm}, \mathrm{RFA}$ has been shown to have similar or superior efficacy to surgical resection, in particular for central HCC. For patients with unresectable early-stage HCC, RFA can lead to curative outcomes and should be recommended as the first-line treatment.

3. MWA is a commonly used thermal ablation method, and no significant differences in local efficacy, incidence of complications and long-term survival have been reported between MWA and RFA. The selection of MWA or RFA should be based on the size and position of tumors, and the operator's experience.

4. PEI is suitable for tumors with a diameter $\leq 3 \mathrm{~cm}$. PEI has good ablative effects and similar long-term efficacy to RFA for tumors with a diameter $\leq 2 \mathrm{~cm}$ despite its higher local recurrence rate compared to RFA. The advantage of PEI is its safety, and in particular, it is suitable for tumors in high-risk locations such as lesions near the hepatic hilar region, gallbladder, and gastrointestinal tracts.

5. Dynamic contrast-enhanced CT or MRI, or contrast-enhanced US, is recommended for assessing the response to ablation around 1 month after ablation therapy.

\section{Transarterial Chemoembolization}

TACE is currently recognized as one of the most commonly used nonsurgical treatments for HCC [150-155] (level 1 evidence).

\section{Basic Principles for TACE}

1. The procedure should be performed under the guidance of a DSA system;

2. The clinical indications must be well understood and strictly followed;

3. Superselective catheterization of the branches of tumor-feeding arteries must be ensured;

4. The patient's liver function must be properly reserved;

\section{Karger ${ }^{\prime \prime}$}


Zhou et al.: 2019 HCC Guidelines in China

5. The procedure must be performed in a standardized and personalized manner;

6. Switching to or combining with other treatments such as surgery, local ablation, systemic treatment and radiation therapy should be considered if the tumor still continues to progress after 3-4 sessions of TACE.

Indications for TACE

1. Patients with CNLC Stage IIb and IIIa HCC and a proportion of patients with Stage IIIb disease, with Child-Pugh Grade A or B and a PS score of 0-2;

2. Patients with resectable CNLC Stage Ib and IIa HCC who are unable or unwilling to receive surgery for other reasons such as older age and severe cirrhosis;

3. Patients with incomplete obstruction of the main portal vein or formation of abundant compensatory collateral branches of the portal vein or recanalized portal vein by portal vein stenting despite complete obstruction;

4. Patients with portal hypertension related bleeding as a result of hepatic artery-portal venous shunt;

5. DSA can be performed for early detection of residual cancer or recurrent lesions following liver resection and TACE can be performed as appropriate.

\section{Contraindications for TACE}

1. Severe liver dysfunction (Child-Pugh Grade C), including jaundice, hepatic encephalopathy, refractory ascites, or hepatorenal syndrome;

2. Serious coagulation dysfunction that cannot be corrected;

3. Complete obstruction of the main portal vein by tumor thrombi, with few collateral branches formed;

4. In the presence of active hepatitis or serious infection that cannot be simultaneously treated;

5. Distal extensive metastasis with an expected survival $<3$ months;

6. Patients with cachexia or multiple organ failure;

7. Tumor burden $>70 \%$ of total liver volume (fractionated embolization with small amounts of lipiodol emulsion and granular embolic agents can be considered in the case of normal liver function);

8. Peripheral white blood cell (WBC) and platelet counts are significantly reduced, with a WBC level $<3.0 \times 10^{9} / \mathrm{L}$ and a platelet level $<50 \times 10^{9} / \mathrm{L}$ (not absolutely contraindicated, e.g., chemotherapy-induced myelosuppression should be excluded in patients with hypersplenism);

9. Renal insufficiency: blood $\mathrm{Cr}>2 \mathrm{mg} / \mathrm{dL}$ or blood $\mathrm{Cr}$ clearance rate $<30 \mathrm{~mL} / \mathrm{min}$.

Overview of Operating Procedures for TACE [156] (level 3 Evidence)

1. Hepatic arteriography is commonly performed using the Seldinger technique from femoral access (radial access can be considered in selected patients). Angiography of the celiac or common hepatic artery should be performed to acquire images in the arterial, parenchymal, and venous phase. Angiography of arteries such as the superior mesenteric artery should be performed to confirm the collateral blood supply. The angiographic manifestations should be carefully analyzed to determine the site, size, number and feeding arteries of tumors.

2. There are 3 broad techniques categorized by the type hepatic arterial chemotherapy and embolization. (1) Transarterial infusion (TAI): chemotherapy drugs are infused through a tumor-feeding artery. Common used chemotherapy drugs for this technique are anthracyclines and platinum doublets. (2) Transarterial embolization (TAE): the feeding arteries of a liver tumor are blocked with embolic agents alone. (3) TACE: a combination of chemo- 
therapy drugs and embolic agents is infused through the tumor-feeding artery. The most commonly used embolic agents for TACE are lipiodol emulsion (containing chemotherapy drugs), standardized gelatin sponge particles, blank microspheres, polyvinyl alcohol particles, and drug-eluting beads. First, a fraction of the chemotherapy drug dose is infused over a period of $\geq 20 \mathrm{~min}$, followed by embolization with the emulsion mixture consisting of the remaining fraction of the chemotherapy drugs and lipiodol. The dose of lipiodol is usually $5-20 \mathrm{~mL}$, but $\leq 30 \mathrm{~mL}$. The treatment stopping boundary is defined by the formation of dense lipiodol deposition in the tumor region and the presence of small portal vein branch shadows around the tumor under fluoroscopic monitoring. Granular embolic agents are used after embolization with lipiodol emulsion. The use of a wellmixed emulsion of ultra-liquified ethiodized oil and chemotherapy drugs is advocated to avoid the embolization of normal liver tissues as a result of agent reflux or the entry of the agents into non-target organs. For embolization, all the tumor-feeding arteries should be embolized to achieve devascularization of the tumor.

Adverse Reactions and Complications of TACE

Post-embolization syndrome is the most common adverse reaction associated with TACE, which mainly manifests as symptoms including fever, pain, nausea, and vomiting. The cause of fever and pain is the ischemia and necrosis of local tissues as a result of hepatic artery embolization, while nausea and vomiting are mainly related to the chemotherapy drugs. In addition, other common adverse reactions may occur including puncture site bleeding, WBC count reduction, transient liver function abnormalities, renal impairment, and dysuria. The adverse reactions usually last 5-7 days and most patients can fully recover from these reactions after symptomatic treatment. Post-TACE complications usually include acute hepatic and renal impairment, gastrointestinal bleeding, cholecystitis, and perforation of the gallbladder, liver abscesses, biloma and ectopic embolization of embolic agents including pulmonary and cerebral lipiodol embolism, perforation of the gastrointestinal tract, spinal cord injury, and diaphragm injury.

Evaluation of Response to TACE

The local response of HCC to TACE should be evaluated in accordance with mRECIST and European Association for the Study of the Liver (EASL) evaluation criteria. The long-term efficacy parameter is overall survival (OS), and the short-term efficacy parameters are objective response rate (ORR) and time to progression (TTP).

Factors that Affect Long-Term Efficacy of TACE [155]

1. Degree of liver cirrhosis and liver function status;

2. Serum AFP level;

3. Tumor volume and burden;

4. Integrity of the tumor capsule;

5. Tumor thrombi in the portal vein;

6. Tumor hypervascularity and blood supply of the tumor;

7. Pathologic subtype;

8. Patient performance status;

9. Serum level of HBV DNA in patients with background of chronic HBV infection.

Follow-Ups and Repeated TACE

Assessment by CT and/or MRI, tumor markers, liver and renal function tests, and blood count are usually recommended 4-6 weeks after the first session of TACE. Repeated sessions of TACE can be postponed if the imaging examination shows thick lipiodol deposition in the

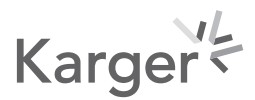


Zhou et al.: 2019 HCC Guidelines in China

liver tumor, necrosis of tumor tissues, and the absence of tumor enlargement and new lesions. The frequency of subsequent TACE should be determined based on the follow-up results, which mainly include the response to previous session of treatment, liver function, and changes in general condition. The follow-ups can be performed at an interval of every 1-3 months or longer. The response of the liver tumor should be evaluated by dynamic contrastenhanced CT and/or MRI to determine the need for repeating TACE. However, 2-4 sessions of TACE are often required for large/huge liver tumors. Combination treatment with TACE plus other treatments is advocated for tumor control, improved quality of life, and long-term survival.

Points Worthy of Notice for TACE

1. Superselective catheterization using a microcatheter is advocated. Catheterization of the tumor-feeding arteries and accurate infusion of lipiodol emulsion and granular embolic agents should be ensured for improved efficacy and protection of liver function.

2. Tumor thrombi in the main portal vein can be effectively managed by portal vein stenting and Iodine-125 seed strips or Iodine-125 seed portal vein stenting [157] (level 2 evidence). The tumor thrombi in the first-order branches of the portal vein can be treated with Iodine-125 seed strips or Iodine-125 seed implantation via direct puncture [158].

3. TACE in combination with local ablation therapy: there are 2 approaches for TACE in combination with thermal ablation therapy. (1) Sequential ablation: TACE followed by local ablation therapy, which are separated by an interval of 1-4 weeks. (2) Concurrent ablation: local ablation therapy is performed during TACE, which results in significantly improved clinical efficacy and reduced hepatic impairment [159] (level 2 evidence). The addition of ablation treatment, as appropriate, to TACE is advocated for improved efficacy.

4. Usage of granular embolic agents, including standardized gelatin sponge particles, polyvinyl alcohol particles, microspheres, drug-eluting beads, etc. Lipiodol emulsion (containing chemotherapy drugs), standardized gelatin sponge particles, blank microspheres, and polyvinyl alcohol particles are often used in conventional TACE (also known as cTACE). Drug-eluting beads (DEBs) are a new type of embolic agent that carries chemotherapy drugs for the treatment of HCC (also known as DEB-TACE). However, no significant differences in overall efficacy have been reported between DEB-TACE and cTACE.

5. Combination of multiple locoregional therapies or with systemic treatment [155]. (1) TACE in combination with local ablation therapy such as RFA, MWA, and cryotherapy [157] (level 2 evidence). (2) TACE in combination with radiation therapy [157] (level 2 evidence). Mainly used as treatments for tumor thrombi in the main portal vein and inferior vena cava and for localized large liver tumors after interventional therapy. (3) TACE in combination with second-stage surgical resection: surgical resection is recommended for large or huge liver tumors which reduce in volume following TACE and become suitable for surgical resection [157] (level 3 evidence). (4) TACE in combination with other treatments, including combination with molecular targeted drugs, immunotherapy, systemic chemotherapy (arsenic trioxide), and radio-immuno-targeted drugs (e.g., 131 I-metuximab). (5) TACE in combination with antiviral therapy: antiviral therapy should be actively performed in combination with TACE in HCC patients with a history of HBV/HCV infection [160] (level 3 evidence).

Summary of main points

1. TACE is one of the most commonly used nonsurgical treatments for HCC.

2. Indications and contraindications of TACE should be noted and followed.

3. Comprehensive angiography should be performed prior to TACE to fully understand the tumor arterial blood supply, including the blood supply of the hepatic artery and ectopic collateral branches. 
4. Superselective catheterization of the branches of tumor-feeding arteries with a microcatheter should be performed for chemoembolization.

5. cTACE is mainly performed with lipiodol emulsion containing chemotherapy drugs, and the combined use with granular embolic agents such as gelatin sponge particles, blank microspheres, and polyvinyl alcohol particles is associated with improved efficacy.

6. Devascularization of the tumor should be achieved to the greatest extent possible. However, attention should be paid to the dose of lipiodol emulsion. The combined use of lipiodol with granular embolic agents is associated with improved efficacy and reduced complications.

7. TACE (including cTACE and DEB-TACE) must be administered based on standardized and individualized regimens.

8. The combination of TACE with other treatment modalities should be advocated for better outcome.

9. HCCs with tumor thrombi in the main trunk and first-order branches of the portal vein can be treated with portal vein stenting in combination with Iodine-125 seed implantation or Iodine-125 seed implantation alone via direct puncture.

\section{Radiation Therapy}

Radiation therapy (abbreviated as radiotherapy) can be categorized into external radiotherapy and internal radiotherapy. External radiotherapy is delivered from outside the body by aiming beams (photons or particle beam radiation) from the radiotherapy device to the tumor. Internal radiotherapy is delivered through the implantation of radionuclides into the tumor through body tracts or needle tracts.

External Radiotherapy

1. Indications for external radiotherapy: (1) CNLC Stage Ia HCC patients and a proportion of patients with Stage Ib HCC. Stereotactic body radiation therapy (SBRT) can be considered as an alternative treatment if surgical resection or local ablation therapy are not clinically indicated or for patients unwilling to receive invasive treatment. SBRT is associated with similar survival rates to surgical resection or local ablation therapy [161-168] (level 2 evidence). (2) Patients with CNLC Stage IIa, IIb, and IIIa HCC; there is evidence that TACE in combination with external radiotherapy is associated with improved local control rate and prolonged survival, as well as better efficacy than monotherapy with TACE or sorafenib or TACE in combination with sorafenib [164, 169-175] (level 2 evidence). (3) Patients with CNLC Stage IIIb HCC; for a proportion of patients with oligometastasis, SBRT can be performed to prolong survival. External radiotherapy can also be used to reduce pain, obstruction, or bleeding caused by lymph node, lung, bone, brain, or adrenal metastasis [176-178] (level 3 evidence). (4) A proportion of patients with initially unresectable HCC will gain the opportunity for surgical resection after tumor shrinkage or downstaging as a result of radiotherapy [178-180] (level 2 evidence). External radiotherapy can also be used as a bridging treatment during the waiting time for liver transplantation. For HCC with a narrow surgical margin $(\leq 1 \mathrm{~cm}$ from the tumor), postoperative adjuvant radiotherapy can reduce the risk of local recurrence or distant metastasis and prolong progression-free survival $[179,180]$ (level 3 evidence).

2. Contraindications for external radiotherapy: external radiotherapy is not recommended in HCC patients with diffusely distributed intrahepatic lesions or CNLC Stage IV HCC.

3. Principles and key points for external radiotherapy: the key principle of performing external radiotherapy for HCC is to comprehensively consider the tumor radiation dose, the dose received by surrounding normal tissues, and the radiotherapy techniques used. 
The key points for performing external radiotherapy for HCC. (1) During preparation of the radiotherapy plan, intrahepatic lesions should be defined by enhanced CT and, if necessary, a wider range of radiographic images such as MRI should be consulted. The regenerative ability of normal liver tissues can be taken into consideration. During radiotherapy, a proportion of normal liver tissue should be preserved without being irradiated to allow reproliferation. (2) The irradiation dose is closely related to survival time and local control rate and is predominantly dependent on the tolerance dose of surrounding normal tissues $[161,181]$. The irradiation dose for HCC is recommended as $\geq 30-60$ Gy in 3 to 6 fractions for SBRT [182], 50-75 Gy for conventional fractionation radiotherapy and $3 \mathrm{~Gy} \times 6$ fractions for neoadjuvant radiotherapy for tumor thrombi in the portal vein [82]. (3) The tolerance of non-tumor tissues is associated with factors including the radiotherapy segmentation method, Child-Pugh classification, normal liver (liver tumor) volume, blood stasis of gastrointestinal tract, and coagulation function. (4) Radiotherapy techniques for HCC; use of 3 dimensional conformal or intensity-modulated radiotherapy, image-guided radiation therapy (IGRT), or SBRT is recommended. IGRT is superior to non-IGRT techniques [161]. Helical tomotherapy is suitable for HCC patients with multiple lesions. Respiratory motion is the main cause of liver tumor motion and deformation during radiotherapy. Multiple techniques can be used to reduce the impact of respiratory motion including respiratory gating techniques, real-time tracking, respiration control, and internal target volume determination techniques based on abdominal compression in combination with 4D CT [183]. (5) Currently, no high-level clinical evidence is available to support the superiority of proton radiotherapy compared with photon radiotherapy for survival rate in patients with HCC [164].

4. The main complications of external radiotherapy: radiation-induced liver diseases (RILDs) are the key dose-limiting complications of external radiotherapy for HCC and can be divided into typical and atypical RILDs. (1) Typical RILDs include increased alkaline phosphatase (AKP) $>2$ times the ULN, jaundice-free ascites, and hepatomegaly. (2) Atypical RILDs include AKP $>2$ times the ULN, ALT $>5$ times the ULN or the pretreatment level, and a reduction of $\geq 2$ points in Child-Pugh Score but with the absence of hepatomegaly and ascites. The diagnosis of RILD must exclude clinical symptoms and liver dysfunction caused by progression of liver tumors, virus activation or drug toxicities [178].

Internal Radiotherapy

Radioactive particle implantation is a method for the local treatment of HCC and includes Y-90 microsphere treatment, iodine-131 monoclonal antibodies, radioactive lipiodol, and iodine-125 seed implantation [37, 176, 177]. Particle implantation techniques include interstitial implantation, portal vein implantation, inferior vena cava implantation, and intrabiliary implantation for treatment of intrahepatic lesions, portal vein tumor thrombi, inferior vena cava tumor thrombi, and intra-bile duct cancer or tumor thrombi, respectively. Strontium chloride $\left({ }^{89} \mathrm{Sr}\right)$ emits $\beta$ rays and can be used for the targeted treatment of bone metastasis from HCC [184] (level 3 evidence).

Summary of main points

1. For patients with CNLC Stage Ia HCC and a proportion of patients with Stage Ib HCC SBRT can be considered as an alternative treatment if surgical resection or local ablation therapy is not clinically indicated or the patients are unwilling to receive invasive treatment.

2. For patients with CNLC Stage IIa, IIb, and IIIa HCC, TACE in combination with external radiotherapy is associated with improved local control rate and prolonged survival and can be used as appropriate. 
3. Patients with CNLC stage IIIb HCC. For selected patients with oligometastasis, SBRT can prolong survival and external radiotherapy can be used to reduce pain, obstruction, or bleeding caused by lymph node, lung, bone, brain, or adrenal metastasis.

4. A proportion of patient will gain the opportunity for surgical resection after tumor shrinkage or downstaging as a result of radiotherapy. External radiotherapy can also be used as a bridging treatment before liver transplantation for HCC or used as adjuvant treatment after resection with a narrow surgical margin.

5. The usual recommended radiation dose is $\geq 30-60$ Gy in 3-6 fractions for stereotactic body radiation therapy, and 50-75 Gy for conventional fractionation radiotherapy. The irradiation dose is closely related to the survival of patients.

6. The dose tolerance of normal liver tissue is associated with radiotherapy segmentation method, Child-Pugh classification, normal liver (liver tumor) volume, blood stasis of gastrointestinal tract, and coagulation function.

7. IGRT is superior to three-dimensional conformal or intensity-modulated radiotherapy. SBRT must be performed under image guidance.

8. Radioactive particle implantation is a method for the local treatment of HCC. Strontium chloride can be used for targeted treatment of bone metastasis from HCC.

\section{Systemic Treatment}

For patients with advanced HCC, effective systemic treatment is associated with reduced tumor burden, improved tumor-related symptoms, improved quality of life, and prolonged survival. Patients can participate in appropriate clinical trials if available systemic treatments fail to produce a satisfactory outcome.

The indications for first- and second-line systemic treatments mainly include (1) CNLC Stage IIIa and IIIb HCC with vascular invasion or extrahepatic metastasis; (2) CNLC Stage IIb HCC with local lesions that are unsuitable for surgical resection or TACE; (3) patients with tumor thrombi in the main portal vein or inferior vena cava; (4) patients with obstructed hepatic blood vessels after multiple sessions of TACE and/or progression after TACE. The relative contraindications mainly include (1) ECOG PS score $>2$, Child-Pugh Score $>7$; (2) moderate and severe bone marrow dysfunction; (3) significant abnormalities in liver and renal function, for example, aminotransferase (AST or ALT) $>5$ times the ULN and/or significantly increased bilirubin $>2$ times the ULN, serum albumin $<28 \mathrm{~g} / \mathrm{L}$ or Cr clearance rate (CCR) $<50 \mathrm{~mL} / \mathrm{min}$; (4) in the presence of infection, fever, active bleeding, or hepatic encephalopathy. Traditional Chinese medicine (TCM) and best supportive care are recommended in patients unable to tolerate or unwilling to receive first- and second-line systemic treatment.

First-Line Treatment

1. Sorafenib: numerous clinical studies have shown that sorafenib provides good survival benefits in patients with advanced HCC in patients from a variety of countries and regions and with different liver disease backgrounds $[185,186]$ (level 1 evidence). The recommended sorafenib dose is $400 \mathrm{mg}$ orally twice daily. Sorafenib can be used in patients with Child-Pugh Grade A and B liver function. Sorafenib provides a more significant survival benefit in patients with Child-Pugh Grade A versus Child-Pugh Class B liver function [187]. Adequate attention should be paid to the potential influence of sorafenib on HBV activation and liver dysfunction and whole-course management of underlying liver diseases is strongly advised. The most common adverse events associated with sorafenib are diarrhea, weight loss, hand-foot syndrome, rash, myocardial ischemia, and hypertension all of which generally occur within 2-6 weeks after the initialization of treatment.

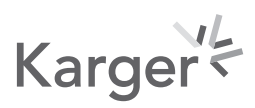


2. Lenvatinib: lenvatinib is recommended for patients with unresectable CNLC Stage IIb, IIIa, and IIIb HCC with Child-Pugh Grade A liver function. A pivotal Phase III clinical trial showed that first-line treatment with lenvatinib is noninferior to sorafenib in efficacy, and provides survival benefits in HBV-related HCC [188] (level 1 evidence). Lenvatinib has been approved for the treatment of patients with advanced HCC and Child-Pugh Grade A liver function. The recommended dose is $12 \mathrm{mg}$ orally per day for patients with a body weight of $\geq 60 \mathrm{~kg}$ or $8 \mathrm{mg}$ per day for patients with a body weight of $<60 \mathrm{~kg}$. Common adverse events associate with lenvatinib include hypertension, diarrhea, anorexia, fatigue, hand-foot syndrome, proteinuria, nausea, and hypothyroidism.

3. Systemic chemotherapy: in China, the FOLFOX4 regimen has been approved for the treatment of locally advanced and metastatic HCCs unsuitable for surgical resection or local treatment $[189,190]$ (level 1 evidence). Furthermore, numerous phase II studies have shown that oxaliplatin-containing systemic chemotherapy in combination with sorafenib is associated with improved objective response rate, prolonged progressionfree survival and overall survival, and a good safety profile [191] (level 3 evidence). This combination therapy can be considered in patients with adequate liver function and good performance status; however, randomized controlled trials are still required to provide high-level evidence to support this approach. In addition, arsenic trioxide has been shown to have a palliative effect on advanced HCC [192] (level 3 evidence), However, hepatorenal toxicity should be monitored and prevented during clinical use.

Second-Line Treatment

1. Regorafenib: regorafenib has been approved for the treatment of patients with CNLC Stage IIb, IIIa, and IIIb HCC who have been previously treated with sorafenib [193] (level 1 evidence). The recommended dose is $160 \mathrm{mg}$ once per day in cycles of 3 weeks on/1 week off treatment. In China, regorafenib can be initiated at a dose of 80 or $120 \mathrm{mg}$ once per day, followed by gradual dose escalation based on the patient's tolerability. The common adverse events are hypertension, hand-foot skin reaction, weakness, and diarrhea.

2. Other second-line treatment regimens: the US FDA has approved the immune-checkpoint inhibitors nivolumab andpembrolizumab for the treatment of patients with HCC who have progressed on previous sorafenib treatment or cannot tolerate sorafenib [194,195] (level 2 evidence). Currently, clinical studies are being conducted to evaluate immunecheckpoint inhibitors developed by Chinese pharmaceutical companies including camrelizumab, toripalimab, and sintilimab. In addition, research is also in progress to investigate combination treatment regimens that employ immunotherapy together with other targeted therapies, chemotherapy drugs and locoregional treatments. Immune-related adverse events (irAEs) can occur in skin, neuroendocrine, gastrointestinal, hepatic, pulmonary, cardiac, and renal systems. Special attention should be paid to serious adverse reactions, including immune enteritis, pneumonia, hepatitis, and myocarditis. In general, moderate or severe irAEs require discontinuation of immune-checkpoint inhibitors and initialization of glucocorticoid immunosuppressive therapy. Management may vary depending on the site and severity of the adverse reactions [196-198]. Other immunomodulators such as interferon- $\alpha$ and thymosin- $\alpha 1[112,199]$ and cellular immunotherapy such as chimeric antigen receptor T (CAR-T) cell therapy [200] and cytokine induced killer (CIK) cell therapy [201] have been shown to have antitumor effects in preliminary studies. However, large clinical trials are still needed for validation of these effects. In addition, the US FDA has approved cabozantinib for patients with progression after first-line systemic treatment [202] (level 1 evidence) and ramucirumab for secondline treatment in patients with a serum AFP level $\geq 400 \mathrm{ng} / \mathrm{mL}[203,204]$ (level 1 
evidence). However, the 2 drugs have not been marketed in China. Clinical studies of second-line treatment with apatinib, a locally developed small molecule anti-angiogenic targeted drug, in HCC patients are under way.

Other Treatments

1. TCM Treatment with TCM can improve clinical symptoms, enhance the immunity of the human body, reduce adverse reactions from radiotherapy and chemotherapy, and improve the quality of life of patients. (1) TCM treatment based on syndrome differentiation [205]: syndrome of liver depression and spleen deficiency, syndrome of liver-gallbladder dampness heat, syndrome of liver heat and blood stasis, syndrome of spleen deficiency and dampness stagnation, and syndrome of liver-kidney yin deficiency. (2)TCM preparations: In addition to using traditional treatment based on syndrome differentiation and oral administration of decoction, the China Food and Drug Administration has approved several modern Chinese herbal preparations such as Huaier granules for adjuvant treatment after surgical resection [206] (level 1 evidence). In addition, Elemene, Cinobufagin, Kanglaite, Kangai, Ganfule Capsule, Jinlong Capsules, Aidi Injection, Brucea Javanidasca oil, and compound Mylabris capsules are used to treat HCC [207-213] (level 4 evidence) with relatively good compliance, safety and tolerability. However, further standardized clinical research is needed to provide high-level evidence-based support for these approaches. (3) Characteristic treatment based on TCM. (A.) Acupuncture treatment: According to the condition of the disease and the actual clinical situation, it is appropriate to apply body acupuncture, scalp acupuncture, electroacupuncture, ear acupuncture, wrist-ankle acupuncture, eye acupuncture, moxibustion, acupoint catgut embedding, acupoint plaster, auricular point pressing with bean, cupping, and other methods. The acupuncture mainly applies on the points of Ganshu and Zusanli (ST36) and is assisted by Yang Lingquan (GB34), Qi Men (LR14), Zhang Men (LV13), San Yinjiao (SP6), etc. For acupoint plaster, the major points are Zhang Men (LIV13), Qi Men (LIV14), Ganshu (BL18), Nei Guan (PC6) and Gong Sun (SP4), and the assisting points, especially for those patients experiencing pain, are Wai Guan (SJ5), Zusanli (ST36), and Yang Lingquan (GB34) and for patients with ascites are Qi Hai (CV6), San Yinjiao (SP6), Yin Lingquan (SP9), etc. (B.) Other treatments: according to the patient's condition, it is appropriate to use traditional Chinese medicine or proprietary Chinese medicine functioning as promoters of blood circulation and to remove blood stasis, clear heat and detoxification for external application treatment, soaking and washing, fumigation and washing, etc.

2. Antiviral treatments and other liver-protecting treatments: for HCC patients with HBV infection, in particular with active replication, oral antiviral treatment with nucleoside (acid) analogs should be performed through the entire duration of treatment for HCC. It is recommended to select potent drugs with a low rate of resistance such as entecavir, tenofovir disoproxil, or tenofovir alafenamide [214] (level 1 evidence). Antiviral treatment with direct-acting antiviral agents (DAAs) or pegylated interferon- $\alpha$ in combination with ribavirin $[215,216]$ are recommended in patients with HCV-related HCC with active hepatitis (level 1 evidence). Abnormal liver function may occur during the natural course of disease and/or treatment in patients with HCC. Therefore, timely and appropriate treatment with liver-protecting drugs is needed. The functions of these drugs are anti-inflammatory, enzyme-reducing, anti-oxidative, detoxifying, cholagogic, and for hepatocyte membrane repair and protection. Liver-protecting drugs include magnesium isoglycyrrhizinate injection, diammonium glycyrrhizinate, compound glycyrrhizin, bicyclol, silymarin, reduced glutathione, ademetionine, ursodeoxycholic acid, polyene phosphatidylcholine, and ulinastatin. These drugs are associated with protection of liver function, increased treatment safety, lower rates of complications, and improved patient quality of life. 
3. Symptomatic supportive treatment: best supportive care should be performed in patients with advanced HCC including analgesic treatment, correction of anemia and hypoalbuminemia, enhanced nutritional support, blood sugar control in patients with diabetes, and management of complications. Common complications include ascites, jaundice, hepatic encephalopathy, gastrointestinal bleeding, and hepatorenal syndrome. Bisphosphonates can be used in patients with bone metastasis. In addition, adequate rehabilitation exercise can increase patient's immunity. Meanwhile, physicians should pay attention to the mental state of their patients and their families and take active measures (including medical therapy) to manage the patient's psychological state and reduce depression and anxiety through palliative treatment and care.

4. Response evaluation for systemic treatment: Currently, response evaluation using RECIST 1.1 is mainly performed in patients receiving systemic treatment. mRECIST can be concurrently used to evaluate treatment response in patients receiving anti-angiogenic therapies. iRECIST can be used to evaluate response in patients receiving treatment with immune-checkpoint inhibitors [217].

Summary of main points

1. Patients with advanced HCC and no contraindications are encouraged to participate in clinical trials.

2. The indications for palliative first- and second-line systemic treatments mainly include (1) patients with CNLC Stage IIIa and IIIb HCC with vascular invasion and/or extrahepatic metastasis; (2) patients with CNLC Stage IIb HCC with local lesions that are unsuitable for surgical resection or TACE; (3) patients with tumor thrombi in the main portal vein or inferior vena cava; (4)patients with obstructed hepatic blood vessels after multiple TACE procedures and/or progression of disease after TACE.

3. Sorafenib, lenvatinib, or oxaliplatin-containing systemic chemotherapy can be selected for first-line treatment.

4. Regorafenib can be selected for second-line treatment.

5. TCM and best supportive care are recommended in HCC patients unable to tolerate or unwilling to receive first- or second-line systemic treatment.

6. Management of underlying liver diseases, including antiviral treatment, liver protection, and cholagogic treatments and supportive symptomatic treatment should be performed as appropriate in addition to antitumor treatment.

\section{Treatment for Rupture of Liver Tumors}

Rupture of liver tumors is a potentially fatal complication of HCC. The in-hospital mortality of simple conservative treatment for a ruptured tumor is extremely high. Therefore, after the success of initial rescue measures, the patient's hemodynamics, liver function, general health status, and possibility of removal of the tumor should be fully evaluated to plan an individualized treatment regimen [218-221].

1. Surgical resection is the first choice in patients with a resectable liver tumor, good liver reserve function, and stable hemodynamics [222, 223] (level 2 evidence).

2. TAE is the first choice in patients with poor liver reserve function and unstable hemodynamics who are unsuitable for surgery [224] (level 4 evidence).

3. In cases where it is not possible to fully evaluate liver function and liver tumors due to limitations of emergency conditions, TAE can be performed first. Corresponding treatment regimens can subsequently be selected based on a follow-up evaluation. Significant survival benefits can be obtained if a second-stage surgical resection is performed [223] (level 2 evidence). 


\section{Acknowledgments}

The authors thank Prof. Mengchao Wu, Prof. Zhaoyou Tang, Prof. Wanyee Lau, Prof. Xiaoping Chen, Prof. Xuehao Wang, Prof. Yan Sun, and Prof. Shusen Zheng for their contribution to the guidelines.

\section{Statement of Ethics}

Our manuscript complies with the guidelines for human studies and was conducted ethically in accordance with the World Medical Association Declaration of Helsinki. In the manuscript, all authors state that subjects have given their written informed consent and that the study protocol was approved by the institute's committee on human research.

The paper is exempt from Ethical Committee approval because the guideline was formulated based on the published data or consensus from the panel members.

\section{Conflict of Interest Statement}

The authors have no conflicts of interest to declare.

\section{Funding Sources}

This research received no external funding.

\section{Author Contributions}

All the authors planned the study and contributed to the interpretation of the data, revisions, and gave input at all stages of the study. All the authors have approved the final version of the manuscript.

\section{References}

1 Torre LA, Bray F, Siegel RL, Ferlay J, Lortet-Tieulent J, Jemal A. Global cancer statistics, 2012. CA Cancer J Clin. 2015 Mar;65(2):87-108.

2 Zhou M, Wang H, Zeng X, Yin P, Zhu J, Chen W, et al. Mortality, morbidity, and risk factors in China and its provinces, 1990-2017: a systematic analysis for the global burden of disease study 2017. Lancet. 2019 Sep 28; 394(10204):1145-58.

3 Zhang BH, Yang BH, Tang ZY. Randomized controlled trial of screening for hepatocellular carcinoma. J Cancer Res Clin Oncol. 2004 Jul;130(7):417-22.

4 Dong Y, Wang WP, Mao F, Ji ZB, Huang BJ. Application of imaging fusion combining contrast-enhanced ultrasound and magnetic resonance imaging in detection of hepatic cellular carcinomas undetectable by conventional ultrasound. J Gastroenterol Hepatol. 2016 Apr;31(4):822-8.

5 da Silva NPB, Hornung M, Beyer LP, Hackl C, Brunner S, Schlitt HJ, et al. Intraoperative shear wave elastography vs. contrast-enhanced ultrasound for the characterization and differentiation of focal liver lesions to optimize liver tumor surgery. Ultraschall Med. 2019 Apr;40(2):205-11.

6 Grgurevic I, Bokun T, Salkic NN, Brkljacic B, Vukelić-Markovic M, Stoos-Veic T, et al. Liver elastography malignancy prediction score for noninvasive characterization of focal liver lesions. Liver Int. 2018 Jun;38(6):105563.

7 Hu H, Han H, Han XK, Wang WP, Ding H. Nomogram for individualised prediction of liver failure risk after hepatectomy in patients with resectable hepatocellular carcinoma: the evidence from ultrasound data. Eur Radiol. 2018 Feb;28(2):877-85.

8 Berzigotti A, Ferraioli G, Bota S, Gilja OH, Dietrich CF. Novel ultrasound-based methods to assess liver disease: the game has just begun. Dig Liver Dis. 2018 Feb;50(2):107-12.

9 Lee YJ, Lee JM, Lee JS, Lee HY, Park BH, Kim YH, et al. Hepatocellular carcinoma: diagnostic performance of multidetector CT and MR imaging-a systematic review and meta-analysis. Radiology. 2015 Apr;275(1): 97-109. 
10 Liu X, Jiang H, Chen J, Zhou Y, Huang Z, Song B. Gadoxetic acid disodium-enhanced magnetic resonance imaging outperformed multidetector computed tomography in diagnosing small hepatocellular carcinoma: a metaanalysis. Liver Transpl. 2017 Dec;23(12):1505-18.

11 Ichikawa T, Saito K, Yoshioka N, Tanimoto A, Gokan T, Takehara Y, et al. Detection and characterization of focal liver lesions: a Japanese phase III, multicenter comparison between gadoxetic acid disodium-enhanced magnetic resonance imaging and contrast-enhanced computed tomography predominantly in patients with hepatocellular carcinoma and chronic liver disease. Invest Radiol. 2010 Mar;45(3):133-41.

12 Ding Y, Chen CZ, Rao SX, Zeng MS. A compared study on Gd+-EOB-DTPA and Gd+-DTPA-enhanced MRI in detection of hepatocellular carcinoma. Chin J Gen Surg. 2013;28(9):682-5. http://dx.doi.org/10.3760/ cma.j.issn.1007-631X.2013.09.010.

13 Yoo SH, Choi JY, Jang JW, Bae SH, Yoon SK, Kim DG, et al. Gd-EOB-DTPA-enhanced MRI is better than MDCT in decision making of curative treatment for hepatocellular carcinoma. Ann Surg Oncol. 2013 Sep;20(9):2893-900.

14 Zeng MS, Ye HY, Guo L, Peng WJ, Lu JP, Teng GJ, et al. Gd-EOB-DTPA-enhanced magnetic resonance imaging for focal liver lesions in Chinese patients: a multicenter, open-label, phase III study. Hepatobiliary Pancreat Dis Int. 2013 Dec;12(6):607-16.

15 Rao SX, Wang J, Wang J, Jiang XQ, Long LL, Li ZP, et al. Chinese consensus on the clinical application of hepatobiliary magnetic resonance imaging contrast agent: gadoxetic acid disodium. J Dig Dis. 2019 Feb;20(2):54-61.

16 Omata M, Cheng AL, Kokudo N, Kudo M, Lee JM, Jia J, et al. Asia-Pacific clinical practice guidelines on the management of hepatocellular carcinoma: a 2017 update. Hepatol Int. 2017 Jul;11(4):317-70.

17 European Association for the Study of the Liver, Electronic address eee, European Association for the Study of the L; European Association for the Study of the Liver.. EASL clinical practice guidelines: management of hepatocellular carcinoma. J Hepatol. 2018 Jul;69(1):182-236. http://dx.doi.org/10.1016/j.jhep.2018.03.019.

18 Marrero JA, Kulik LM, Sirlin CB, Zhu AX, Finn RS, Abecassis MM, et al. Diagnosis, staging, and management of hepatocellular carcinoma: 2018 practice guidance by the American association for the study of liver diseases. Hepatology. 2018 Aug;68(2):723-50.

19 Cho ES, Choi JY. MRI features of hepatocellular carcinoma related to biologic behavior. Korean J Radiol. 2015 May-Jun;16(3):449-64.

20 Hwang J, Kim YK, Jeong WK, Choi D, Rhim H, Lee WJ. Nonhypervascular hypointense nodules at gadoxetic acidenhanced MR imaging in chronic liver disease: diffusion-weighted imaging for characterization. Radiology. 2015 Oct;276(1):137.

21 Renzulli M, Biselli M, Brocchi S, Granito A, Vasuri F, Tovoli F, et al. New hallmark of hepatocellular carcinoma, early hepatocellular carcinoma and high-grade dysplastic nodules on Gd-EOB-DTPA MRI in patients with cirrhosis: a new diagnostic algorithm. Gut. 2018 Sep;67(9):1674-82.

22 Kitao A, Zen Y, Matsui O, Gabata T, Kobayashi S, Koda W, et al. Hepatocellular carcinoma: signal intensity at gadoxetic acid-enhanced MR Imaging: correlation with molecular transporters and histopathologic features. Radiology. 2010 Sep;256(3):817-26.

23 Kitao A, Matsui O, Yoneda N, Kozaka K, Kobayashi S, Sanada J, et al. Hepatocellular carcinoma with $\beta$-catenin mutation: imaging and pathologic characteristics. Radiology. 2015 Jun;275(3):708-17. http://dx.doi. org/10.1148/radiol.14141315.

24 Lei Z, Li J, Wu D, Xia Y, Wang Q, Si A, et al. Nomogram for preoperative estimation of microvascular invasion risk in hepatitis B virus-related hepatocellular carcinoma within the Milan criteria. JAMA Surg. 2016 Apr; 151(4):356-63.

25 Peng J, Zhang J, Zhang Q, Xu Y, Zhou J, Liu L. A radiomics nomogram for preoperative prediction of microvascular invasion risk in hepatitis B virus-related hepatocellular carcinoma. Diagn Interv Radiol. 2018 May-Jun; 24(3):121-7.

26 Xu X, Zhang HL, Liu QP, Sun SW, Zhang J, Zhu FP, et al. Radiomic analysis of contrast-enhanced CT predicts microvascular invasion and outcome in hepatocellular carcinoma. J Hepatol. 2019 Jun;70(6):1133-44.

27 Park JW, Kim JH, Kim SK, Kang KW, Park KW, Choi JI, et al. A prospective evaluation of 18F-FDG and 11C-acetate PET/CT for detection of primary and metastatic hepatocellular carcinoma. J Nucl Med. 2008 Dec;49(12): 1912-21.

28 Lin CY, Chen JH, Liang JA, Lin CC, Jeng LB, Kao CH. 18F-FDG PET or PET/CT for detecting extrahepatic metastases or recurrent hepatocellular carcinoma: a systematic review and meta-analysis. Eur J Radiol. 2012 Sep; 81(9):2417-22.

29 Boellaard R, O’Doherty MJ, Weber WA, Mottaghy FM, Lonsdale MN, Stroobants SG, et al. FDG PET and PET/CT: EANM procedure guidelines for tumour PET imaging: version 1.0. Eur J Nucl Med Mol Imaging. 2010 Jan;37(1): 181-200.

30 Boellaard R, Delgado-Bolton R, Oyen WJ, Giammarile F, Tatsch K, Eschner W, et al. FDG PET/CT: EANM procedure guidelines for tumour imaging: version 2.0. Eur J Nucl Med Mol Imaging. 2015 Feb;42(2):328-54.

31 Wahl RL, Jacene H, Kasamon Y, Lodge MA. From RECIST to PERCIST: evolving considerations for PET response criteria in solid tumors. J Nucl Med. 2009 May;50(Suppl 1):122S-50S.

32 Chalian H, Töre HG, Horowitz JM, Salem R, Miller FH, Yaghmai V. Radiologic assessment of response to therapy: comparison of RECIST versions 1.1 and 1.0. Radiographics. 2011 Nov-Dec;31(7):2093-105.

33 Ferda J, Ferdová E, Baxa J, Kreuzberg B, Daum O, Třeška V, et al. The role of 18F-FDG accumulation and arterial enhancement as biomarkers in the assessment of typing, grading and staging of hepatocellular carcinoma using 18F-FDG-PET/CT with integrated dual-phase CT angiography. Anticancer Res. 2015 Apr;35(4):2241-6. 


\begin{tabular}{l|l|}
\hline Liver Cancer 2020;9:682-720 \\
\hline DOI: 10.1159/000509424 & $\begin{array}{l}\text { @ 2020 The Author(s). Published by S. Karger AG, Basel } \\
\text { www.karger.com/lic }\end{array}$ \\
\hline
\end{tabular}

Zhou et al.: 2019 HCC Guidelines in China

34 Hyun SH, Eo JS, Lee JW, Choi JY, Lee KH, Na SJ, et al. Prognostic value of (18)F-fluorodeoxyglucose positron emission tomography/computed tomography in patients with Barcelona clinic liver cancer stages 0 and $\mathrm{A}$ hepatocellular carcinomas: a multicenter retrospective cohort study. Eur J Nucl Med Mol Imaging. 2016 Aug; 43(9):1638-45.

35 Lee JW, Oh JK, Chung YA, Na SJ, Hyun SH, Hong IK, et al. Prognostic significance of 18F-FDG uptake in hepatocellular carcinoma treated with transarterial chemoembolization or concurrent chemoradiotherapy: a multicenter retrospective cohort study. J Nucl Med. 2016 Apr;57(4):509-16. http://dx.doi.org/10.2967/ jnumed.115.167338.

36 Na SJ, Oh JK, Hyun SH, Lee JW, Hong IK, Song BI, et al. 18F-FDG PET/CT can predict survival of advanced hepatocellular carcinoma patients: a multicenter retrospective cohort study. J Nucl Med. 2017 May;58(5):730-6. http://dx.doi.org/10.2967/jnumed.116.182022.

37 Cheung TT, Ho CL, Lo CM, Chen S, Chan SC, Chok KS, et al. 11C-acetate and 18F-FDG PET/CT for clinical staging and selection of patients with hepatocellular carcinoma for liver transplantation on the basis of Milan criteria: surgeon's perspective. J Nucl Med. 2013 Feb;54(2):192-200.

38 Bertagna F, Bertoli M, Bosio G, Biasiotto G, Sadeghi R, Giubbini R, et al. Diagnostic role of radiolabelled choline PET or PET/CT in hepatocellular carcinoma: a systematic review and meta-analysis. Hepatol Int. 2014 Oct; 8(4):493-500.

39 Zhang Y, Shi H, Cheng D, Jiang L, Xiu Y, Li B, et al. Added value of SPECT/spiral CT versus SPECT in diagnosing solitary spinal lesions in patients with extraskeletal malignancies. Nucl Med Commun. 2013 May;34(5):451-8.

40 Hectors SJ, Wagner M, Besa C, Huang W, Taouli B. Multiparametric FDG-PET/MRI of hepatocellular carcinoma: initial experience. Contrast Media Mol Imaging. 2018;2018:5638283.

41 Forner A, Vilana R, Ayuso C, Bianchi L, Solé M, Ayuso JR, et al. Diagnosis of hepatic nodules 20 mm or smaller in cirrhosis: prospective validation of the noninvasive diagnostic criteria for hepatocellular carcinoma. Hepatology. 2008 Jan;47(1):97-104.

42 Roberts LR, Sirlin CB, Zaiem F, Almasri J, Prokop LJ, Heimbach JK, et al. Imaging for the diagnosis of hepatocellular carcinoma: a systematic review and meta-analysis. Hepatology. 2018 Jan;67(1):401-21.

43 Paradish V, Fukayama M, Park YN, Schirmacher P. Tumors of the liver and intrahepatic bile ducts. WHO Classification of tumours of the digestive system tumours. Lyon, France: International Agency for Research on Cancer; 2019.

44 Zhou J, Yu L, Gao X, Hu J, Wang J, Dai Z, et al. Plasma microRNA panel to diagnose hepatitis B virus-related hepatocellular carcinoma. J Clin Oncol. 2011 Dec 20;29(36):4781-8.

45 Zhou J, Huang A, Yang XR. Liquid biopsy and its potential for management of hepatocellular carcinoma. J Gastrointest Cancer. 2016 Jun; 47(2):157-67.

46 Westra WH, Hruban RH, Phelps TH, Isacson C. Surgical pathology dissection: an illustrated guide. New York: Springer; 2003.

47 Nara S, Shimada K, Sakamoto Y, Esaki M, Kishi Y, Kosuge T, et al. Prognostic impact of marginal resection for patients with solitary hepatocellular carcinoma: evidence from 570 hepatectomies. Surgery. 2012 Apr;151(4): 526-36.

48 Wenming C. Surgical pathology of liver and gallbaldder tumors. Beijing: People's Medical Publishing House; 2015.

49 Torbenson MS, Ng IOL, Park YN, Roncalli M, Sakamato M. WHO Classification of tumours of the digestive system tumours. Lyon, France: International Agency for Research on Cancer; 2019. p. 229-39.

50 Scheuer PJ. Classification of chronic viral hepatitis: a need for reassessment. J Hepatol. 1991 Nov;13(3):372-4.

51 Society of Infectious Diseases C, Chinese Society of Hepatology C; Chinese Society of Hepatology, Chinese Medical Association.. Regimens for prevention and treatment of viral hepatitis. Chin J Infect Dis. 2001;19(1): 56-62. http://dx.doi.org/10.3760/j.issn:1000-6680.2001.01.027.

52 Lasser C. Exosomes in diagnostic and therapeutic applications: biomarker, vaccine and RNA interference delivery vehicle. Expert Opin Biol Ther. 2015 Jan;15(1):103-17.

53 Rodríguez-Perálvarez M, Luong TV, Andreana L, Meyer T, Dhillon AP, Burroughs AK. A systematic review of microvascular invasion in hepatocellular carcinoma: diagnostic and prognostic variability. Ann Surg Oncol. 2013 Jan;20(1):325-39. http://dx.doi.org/10.1245/s10434-012-2513-1.

54 Association PSoLcCA, Association LCGSoLDCM, Association PSoPCA; Liver Cancer Study Group, Chinese Society of Hepatology, Chinese Medical Association; Chinese Societies of Pathology, Chinese Anti-Cancer Association.. Evidence-based practice guidelines for the standardized pathological diagnosis of primary liver cancer(2015 edition). Chin J Hepatobiliary Surg. 2015;21(3):145-51. http://dx.doi.org/10.3760/ cma.j.issn.1007-8118.2015.03.001.

55 Eguchi S, Takatsuki M, Hidaka M, Soyama A, Tomonaga T, Muraoka I, et al. Predictor for histological microvascular invasion of hepatocellular carcinoma: a lesson from 229 consecutive cases of curative liver resection. World J Surg. 2010 May;34(5):1034-8.

56 Fujita N, Aishima S, Iguchi T, Mano Y, Taketomi A, Shirabe K, et al. Histologic classification of microscopic portal venous invasion to predict prognosis in hepatocellular carcinoma. Hum Pathol. 2011 Oct;42(10): 1531-8.

57 Iguchi T, Shirabe K, Aishima S, Wang H, Fujita N, Ninomiya M, et al. New pathologic stratification of microvascular invasion in hepatocellular carcinoma: predicting prognosis after living-donor liver transplantation. Transplantation. 2015 Jun; 99(6):1236-42. 
58 Shi L, Zhang SJ, Chen J, Lu SX, Fan XJ, Tong JH, et al. A comparability study of immunohistochemical assays for PD-L1 expression in hepatocellular carcinoma. Mod Pathol. 2019 Nov;32(11):1646-56.

59 Wen T, Jin C, Facciorusso A, Donadon M, Han HS, Mao Y, et al. Multidisciplinary management of recurrent and metastatic hepatocellular carcinoma after resection: an international expert consensus. Hepatobiliary Surg Nutr. 2018 Oct; 7(5):353-71.

60 Xie DY, Fan HK, Ren ZG, Fan J, Gao Q. Identifying clonal origin of multifocal hepatocellular carcinoma and its clinical implications. Clin Transl Gastroenterol. 2019 Feb;10(2):e00006.

61 Wang B, Xia CY, Lau WY, Lu XY, Dong H, Yu WL, et al. Determination of clonal origin of recurrent hepatocellular carcinoma for personalized therapy and outcomes evaluation: a new strategy for hepatic surgery. J Am Coll Surg. 2013 Dec;217(6):1054-62.

62 Cong WM, Wu MC. New insights into molecular diagnostic pathology of primary liver cancer: advances and challenges. Cancer Lett. 2015 Nov 1;368(1):14-9.

63 Bruix J, Castells A, Bosch J, Feu F, Fuster J, Garcia-Pagan JC, et al. Surgical resection of hepatocellular carcinoma in cirrhotic patients: prognostic value of preoperative portal pressure. Gastroenterology. 1996 Oct;111(4): 1018-22.

64 Kubota K, Makuuchi M, Kusaka K, Kobayashi T, Miki K, Hasegawa K, et al. Measurement of liver volume and hepatic functional reserve as a guide to decision-making in resectional surgery for hepatic tumors. Hepatology. 1997 Nov;26(5):1176-81.

65 Imamura H, Seyama Y, Kokudo N, Maema A, Sugawara Y, Sano K, et al. One thousand fifty-six hepatectomies without mortality in 8 years. Arch Surg. 2003 Nov;138(11):1198-206.

66 Cescon M, Colecchia A, Cucchetti A, Peri E, Montrone L, Ercolani G, et al. Value of transient elastography measured with FibroScan in predicting the outcome of hepatic resection for hepatocellular carcinoma. Ann Surg. 2012 Nov;256(5):706-3.

67 Rajakannu M, Cherqui D, Ciacio O, Golse N, Pittau G, Allard MA, et al. Liver stiffness measurement by transient elastography predicts late posthepatectomy outcomes in patients undergoing resection for hepatocellular carcinoma. Surgery. 2017 Oct;162(4):766-74.

68 Shen Y, Zhou C, Zhu G, Shi G, Zhu X, Huang C, et al. Liver stiffness assessed by shear wave elastography predicts postoperative liver failure in patients with hepatocellular carcinoma. J Gastrointest Surg. 2017 Sep;21(9): 1471-9.

69 Zhong JH, Ke Y, Gong WF, Xiang BD, Ma L, Ye XP, et al. Hepatic resection associated with good survival for selected patients with intermediate and advanced-stage hepatocellular carcinoma. Ann Surg. 2014 Aug; 260(2):329-40.

70 Xiao H, Zhang B, Mei B, Zuo C, Wei G, Wang R, et al. Hepatic resection for hepatocellular carcinoma in patients with portal hypertension: a long-term benefit compared with transarterial chemoembolization and thermal ablation. Medicine. 2015 Feb;94(7):e495.

71 Bosch J, Abraldes JG, Berzigotti A, García-Pagan JC. The clinical use of HVPG measurements in chronic liver disease. Nat Rev Gastroenterol Hepatol. 2009 Oct;6(10):573-82.

72 Chen X, Zhai J, Cai X, Zhang Y, Wei L, Shi L, et al. Severity of portal hypertension and prediction of postoperative liver failure after liver resection in patients with Child-Pugh grade A cirrhosis. Br J Surg. 2012 Dec;99(12): 1701-10.

73 Mohkam K, Dumont PN, Manichon AF, Jouvet JC, Boussel L, Merle P, et al. No-touch multibipolar radiofrequency ablation vs. surgical resection for solitary hepatocellular carcinoma ranging from 2 to $5 \mathrm{~cm}$. J Hepatol. 2018 Jun;68(6):1172-80. http://dx.doi.org/10.1016/j.jhep.2018.01.014.

74 Chen MS, Li JQ, Zheng Y, Guo RP, Liang HH, Zhang YQ, et al. A prospective randomized trial comparing percutaneous local ablative therapy and partial hepatectomy for small hepatocellular carcinoma. Ann Surg. 2006 Mar;243(3):321-8.

75 Feng K, Yan J, Li X, Xia F, Ma K, Wang S, et al. A randomized controlled trial of radiofrequency ablation and surgical resection in the treatment of small hepatocellular carcinoma. J Hepatol. 2012 Oct;57(4):794-802.

76 Xu Q, Kobayashi S, Ye X, Meng X. Comparison of hepatic resection and radiofrequency ablation for small hepatocellular carcinoma: a meta-analysis of 16,103 patients. Sci Rep. 2014 Nov 28;4:7252.

77 Liu PH, Hsu CY, Hsia CY, Lee YH, Huang YH, Chiou YY, et al. Surgical resection versus radiofrequency ablation for single hepatocellular carcinoma $\leq 2 \mathrm{~cm}$ in a propensity score model. Ann Surg. 2016 Mar;263(3):538-45.

78 Torzilli G, Belghiti J, Kokudo N, Takayama T, Capussotti L, Nuzzo G, et al. A snapshot of the effective indications and results of surgery for hepatocellular carcinoma in tertiary referral centers: is it adherent to the EASL/ AASLD recommendations?: an observational study of the HCC East-West study group. Ann Surg. 2013 May; 257(5):929-37.

79 Yin L, Li H, Li AJ, Lau WY, Pan ZY, Lai EC, et al. Partial hepatectomy vs. transcatheter arterial chemoembolization for resectable multiple hepatocellular carcinoma beyond Milan criteria: a RCT. J Hepatol. 2014 Jul; 61(1):82-8.

80 Shi J, Lai EC, Li N, Guo WX, Xue J, Lau WY, et al. Surgical treatment of hepatocellular carcinoma with portal vein tumor thrombus. Ann Surg Oncol. 2010 Aug;17(8):2073-80.

81 Wang K, Guo WX, Chen MS, Mao YL, Sun BC, Shi J, et al. Multimodality treatment for hepatocellular carcinoma with portal vein tumor thrombus: a large-scale, multicenter, propensity mathching score analysis. Medicine. 2016 Mar;95(11):e3015. 


\begin{tabular}{l|l|}
\hline Liver Cancer 2020;9:682-720 \\
\hline DOI: 10.1159/000509424 & $\begin{array}{l}\text { @ 2020 The Author(s). Published by S. Karger AG, Basel } \\
\text { www.karger.com/lic }\end{array}$ \\
\hline
\end{tabular}

Zhou et al.: 2019 HCC Guidelines in China

82 Wei X, Jiang Y, Zhang X, Feng S, Zhou B, Ye X, et al. Neoadjuvant three-dimensional conformal radiotherapy for resectable hepatocellular carcinoma with portal vein tumor thrombus: a randomized, open-label, multicenter controlled study. J Clin Oncol. 2019 Aug 20;37(24):2141-51.

83 Li XL, Zhu XD, Cai H, Li Y, Zhou J, Fan J, et al. Postoperative $\alpha$-fetoprotein response predicts tumor recurrence and survival after hepatectomy for hepatocellular carcinoma: A propensity score matching analysis. Surgery. 2019 Jun;165(6):1161-7. http://dx.doi.org/10.1016/j.surg.2019.01.009.

84 Jiang HT, Cao JY. Impact of laparoscopic versus open hepatectomy on perioperative clinical outcomes of patients with primary hepatic carcinoma. Chin Med Sci J. 2015 Jun;30(2):80-3.

85 Wu X, Huang Z, Lau WY, Li W, Lin P, Zhang L, et al. Perioperative and long-term outcomes of laparoscopic versus open liver resection for hepatocellular carcinoma with well-preserved liver function and cirrhotic background: a propensity score matching study. Surg Endosc. 2019 Jan;33(1):206-15.

86 Cheung TT, Han HS, She WH, Chen KH, Chow PKH, Yoong BK, et al. The Asia Pacific consensus statement on laparoscopic liver resection for hepatocellular carcinoma: a report from the 7th Asia-Pacific primary liver cancer expert meeting held in Hong Kong. Liver Cancer. 2018 Mar;7(1):28-39.

87 Shi M, Guo RP, Lin XJ, Zhang YQ, Chen MS, Zhang CQ, et al. Partial hepatectomy with wide versus narrow resection margin for solitary hepatocellular carcinoma: a prospective randomized trial. Ann Surg. 2007 Jan; 245(1):36-43.

88 Yang P, Si A, Yang J, Cheng Z, Wang K, Li J, et al. A wide-margin liver resection improves long-term outcomes for patients with HBV-related hepatocellular carcinoma with microvascular invasion. Surgery. 2019 Apr; 165(4):721-30.

89 Liu CL, Fan ST, Lo CM, Tung-Ping Poon R, Wong J. Anterior approach for major right hepatic resection for large hepatocellular carcinoma. Ann Surg. 2000 Jul;232(1):25-31.

90 Zhou C, Peng Y, Zhou K, Zhang L, Zhang X, Yu L, et al. Surgical resection plus radiofrequency ablation for the treatment of multifocal hepatocellular carcinoma. Hepatobiliary Surg Nutr. 2019 Feb;8(1):19-28.

91 Zhang ZM, Lai EC, Zhang C, Yu HW, Liu Z, Wan BJ, et al. The strategies for treating primary hepatocellular carcinoma with portal vein tumor thrombus. Int J Surg. 2015 Aug;20:8-16.

92 Fu SY, Lau WY, Li AJ, Yang Y, Pan ZY, Sun YM, et al. Liver resection under total vascular exclusion with or without preceding Pringle manoeuvre. Br J Surg. 2010 Jan;97(1):50-5.

93 Satoh S, Ikai I, Honda G, Okabe H, Takeyama O, Yamamoto Y, et al. Clinicopathologic evaluation of hepatocellular carcinoma with bile duct thrombi. Surgery. 2000 Nov;128(5):779-83.

94 Tang ZY, Uy YQ, Zhou XD, Ma ZC, Lu JZ, Lin ZY, et al. Cytoreduction and sequential resection for surgically verified unresectable hepatocellular carcinoma: evaluation with analysis of 72 patients. World J Surg. 1995 Nov-Dec;19(6):784-9.

95 Tang ZY, Yu YQ, Zhou XD, Ma ZC, Yang BH, Lin ZY, et al. Treatment of unresectable primary liver cancer: with reference to cytoreduction and sequential resection. World J Surg. 1995 Jan-Feb;19(1):47-52.

96 Wakabayashi H, Okada S, Maeba T, Maeta H. Effect of preoperative portal vein embolization on major hepatectomy for advanced-stage hepatocellular carcinomas in injured livers: a preliminary report. Surg Today. 1997;27(5):403-10.

97 Ogata S, Belghiti J, Farges O, Varma D, Sibert A, Vilgrain V. Sequential arterial and portal vein embolizations before right hepatectomy in patients with cirrhosis and hepatocellular carcinoma. Br J Surg. 2006 Sep;93(9): 1091-8.

98 Wang Z, Peng Y, Hu J, Wang X, Sun H, Sun J, et al. Associating liver partition and portal vein ligation for staged hepatectomy for unresectable hepatitis B virus-related hepatocellular carcinoma: a single center study of 45 patients. Ann Surg. 2020;271(3):534-41. http://dx.doi.org/10.1097/SLA.0000000000002942.

99 D’Haese JG, Neumann J, Weniger M, Pratschke S, Björnsson B, Ardiles V, et al. Should ALPPS be used for liver resection in intermediate-stage HCC? Ann Surg Oncol. 2016 Apr;23(4):1335-43.

100 Zheng S, Li J, Xiao L, Cao L, Guo P. Totally laparoscopic associating liver partition and portal vein ligation for staged hepatectomy for the treatment of cirrhotic hepatocellular carcinoma. Chinese J Dig Surg. 2014;13(7): 502-7. http://dx.doi.org/10.3760/cma.j.issn.1673-9752.2014.07.002.

101 Hong de F, Zhang YB, Peng SY, Huang DS. Percutaneous microwave ablation liver partition and portal vein embolization for rapid liver regeneration: a minimally invasive first step of ALPPS for hepatocellular carcinoma. Ann Surg. 2016 Jul;264(1):e1-2. http://dx.doi.org/10.1097/SLA.0000000000001707.

102 Zhou WP, Lai EC, Li AJ, Fu SY, Zhou JP, Pan ZY, et al. A prospective, randomized, controlled trial of preoperative transarterial chemoembolization for resectable large hepatocellular carcinoma. Ann Surg. 2009 Feb;249(2): 195-202.

103 Shi HY, Wang SN, Wang SC, Chuang SC, Chen CM, Lee KT. Preoperative transarterial chemoembolization and resection for hepatocellular carcinoma: a nationwide Taiwan database analysis of long-term outcome predictors. J Surg Oncol. 2014 Apr;109(5):487-93.

104 Yin J, Li N, Han Y, Xue J, Deng Y, Shi J, et al. Effect of antiviral treatment with nucleotide/nucleoside analogs on postoperative prognosis of hepatitis B virus-related hepatocellular carcinoma: a two-stage longitudinal clinical study. J Clin Oncol. 2013 Oct 10;31(29):3647-55.

105 Huang G, Lau WY, Wang ZG, Pan ZY, Yuan SX, Shen F, et al. Antiviral therapy improves postoperative survival in patients with hepatocellular carcinoma: a randomized controlled trial. Ann Surg. 2015 Jan;261(1):56-66. 
106 Wang Z, Ren Z, Chen Y, Hu J, Yang G, Yu L, et al. Adjuvant transarterial chemoembolization for HBV-related hepatocellular carcinoma after resection: a randomized controlled study. Clin Cancer Res. 2018 May 1;24(9): 2074-81.

107 Wei W, Jian PE, Li SH, Guo ZX, Zhang YF, Ling YH, et al. Adjuvant transcatheter arterial chemoembolization after curative resection for hepatocellular carcinoma patients with solitary tumor and microvascular invasion: a randomized clinical trial of efficacy and safety. Cancer Commun. 2018 Oct 10;38(1):61.

108 Chen Q, Shu C, Laurence AD, Chen Y, Peng BG, Zhen ZJ, et al. Effect of Huaier granule on recurrence after curative resection of HCC: a multicentre, randomised clinical trial. Gut. 2018 Nov;67(11):2006-16.

109 Huang G, Li PP, Lau WY, Pan ZY, Zhao LH, Wang ZG, et al. Antiviral therapy reduces hepatocellular carcinoma recurrence in patients with low HBV-DNA levels: a randomized controlled trial. Ann Surg. 2018 Dec;268(6): 943-54.

110 Fan J, Zhou J, Wu ZQ, Qiu SJ, Wang XY, Shi YH, et al. Efficacy of different treatment strategies for hepatocellular carcinoma with portal vein tumor thrombosis. World J Gastroenterol. 2005 Feb 28;11(8):1215-9.

111 Nishiguchi S, Tamori A, Kubo S. Effect of long-term postoperative interferon therapy on intrahepatic recurrence and survival rate after resection of hepatitis $\mathrm{C}$ virus-related hepatocellular carcinoma. Intervirology. 2005;48(1):71-5.

112 Sun HC, Tang ZY, Wang L, Qin LX, Ma ZC, Ye QH, et al. Postoperative interferon alpha treatment postponed recurrence and improved overall survival in patients after curative resection of HBV-related hepatocellular carcinoma: a randomized clinical trial. J Cancer Res Clin Oncol. 2006 Jul;132(7):458-65.

113 Lo CM, Liu CL, Chan SC, Lam CM, Poon RT, Ng IO, et al. A randomized, controlled trial of postoperative adjuvant interferon therapy after resection of hepatocellular carcinoma. Ann Surg. 2007 Jun;245(6):831-42.

114 Mazzaferro V, Romito R, Schiavo M, Mariani L, Camerini T, Bhoori S, et al. Prevention of hepatocellular carcinoma recurrence with alpha-interferon after liver resection in HCV cirrhosis. Hepatology. 2006 Dec; 44(6):1543-54.

115 Ji J, Shi J, Budhu A, Yu Z, Forgues M, Roessler S, et al. MicroRNA expression, survival, and response to interferon in liver cancer. $N$ Engl J Med. 2009 Oct 8;361(15):1437-47.

116 Bruix J, Takayama T, Mazzaferro V, Chau GY, Yang J, Kudo M, et al. Adjuvant sorafenib for hepatocellular carcinoma after resection or ablation (STORM): a phase 3, randomised, double-blind, placebo-controlled trial. Lancet Oncol. 2015 Oct;16(13):1344-54.

117 Wang SN, Chuang SC, Lee KT. Efficacy of sorafenib as adjuvant therapy to prevent early recurrence of hepatocellular carcinoma after curative surgery: a pilot study. Hepatol Res. 2014 May;44(5):523-31.

118 Sapisochin G, Bruix J. Liver transplantation for hepatocellular carcinoma: outcomes and novel surgical approaches. Nat Rev Gastroenterol Hepatol. 2017 Apr;14(4):203-17.

119 Fan ST. Selection of HCC patients for liver transplantation: the Milan criteria, Hangzhou criteria and beyond. Hepatobiliary Pancreat Dis Int. 2008 Jun;7(3):233-4.

120 Fan J, Yang GS, Fu ZR, Peng ZH, Xia Q, Peng CH, et al. Liver transplantation outcomes in 1,078 hepatocellular carcinoma patients: a multi-center experience in Shanghai, China. J Cancer Res Clin Oncol. 2009 Oct;135(10): 1403-12.

121 Li J, Yan LN, Yang J, Chen ZY, Li B, Zeng Y, et al. Indicators of prognosis after liver transplantation in Chinese hepatocellular carcinoma patients. World J Gastroenterol. 2009 Sep 7;15(33):4170-6.

122 Shao Z, Yang GS, Yang N, Zhang HB. Application of Sanya criteria in the treatment of liver transplantation for hepatocellular carcinoma. Chin J Pract Surg. 2008;28(6):466-9. http://dx.doi.org/10.3321/j.issn:10052208.2008.06.018.

123 Llovet JM, Pavel M, Rimola J, Diaz MA, Colmenero J, Saavedra-Perez D, et al. Pilot study of living donor liver transplantation for patients with hepatocellular carcinoma exceeding Milan criteria (Barcelona clinic liver cancer extended criteria). Liver Transpl. 2018 Mar;24(3):369-79.

124 Pinheiro RS, Waisberg DR, Nacif LS, Rocha-Santos V, Arantes RM, Ducatti L, et al. Living donor liver transplantation for hepatocellular cancer: an (almost) exclusive Eastern procedure? Transl Gastroenterol Hepatol. 2017;2:68.

125 Sposito C, Cucchetti A, Mazzaferro V. Assessing competing risks for death following liver transplantation for hepatocellular carcinoma. Dig Dis Sci. 2019 Apr;64(4):1001-7.

126 Segev DL, Sozio SM, Shin EJ, Nazarian SM, Nathan H, Thuluvath PJ, et al. Steroid avoidance in liver transplantation: meta-analysis and meta-regression of randomized trials. Liver Transpl. 2008 Apr;14(4):512-25.

127 Rodríguez-Perálvarez M, Tsochatzis E, Naveas MC, Pieri G, García-Caparrós C, O’Beirne J, et al. Reduced exposure to calcineurin inhibitors early after liver transplantation prevents recurrence of hepatocellular carcinoma. J Hepatol. 2013 Dec;59(6):1193-9. http://dx.doi.org/10.1016/j.jhep.2013.07.012.

128 Zhou J, Wang Z, Wu ZQ, Qiu SJ, Yu Y, Huang XW, et al. Sirolimus-based immunosuppression therapy in liver transplantation for patients with hepatocellular carcinoma exceeding the Milan criteria. Transplant Proc. 2008 Dec;40(10):3548-53.

129 Liang W, Wang D, Ling X, Kao AA, Kong Y, Shang Y, et al. Sirolimus-based immunosuppression in liver transplantation for hepatocellular carcinoma: a meta-analysis. Liver Transpl. 2012 Jan;18(1):62-9.

130 Geissler EK, Schnitzbauer AA, Zülke C, Lamby PE, Proneth A, Duvoux C, et al. Sirolimus use in liver transplant recipients with hepatocellular carcinoma: a randomized, multicenter, open-label phase 3 trial. Transplantation. 2016 Jan;100(1):116-25. 


\begin{tabular}{l|l|}
\hline Liver Cancer 2020;9:682-720 \\
\hline DOI: 10.1159/000509424 & $\begin{array}{l}\text { @ 2020 The Author(s). Published by S. Karger AG, Basel } \\
\text { www.karger.com/lic }\end{array}$ \\
\hline
\end{tabular}

Zhou et al.: 2019 HCC Guidelines in China

131 Thorat A, Jeng LB, Yang HR, Yeh CC, Hsu SC, Chen TH, et al. Assessing the role of everolimus in reducing hepatocellular carcinoma recurrence after living donor liver transplantation for patients within the UCSF criteria: re-inventing the role of mammalian target of rapamycin inhibitors. Ann Hepatobiliary Pancreat Surg. 2017 Nov;21(4):205-11.

132 Filgueira NA. Hepatocellular carcinoma recurrence after liver transplantation: risk factors, screening and clinical presentation. World J Hepatol. 2019 Mar 27;11(3):261-72.

$133 \mathrm{Au} \mathrm{KP}$, Chok KSH. Multidisciplinary approach for post-liver transplant recurrence of hepatocellular carcinoma: a proposed management algorithm. World J Gastroenterol. 2018 Dec 7;24(45):5081-94.

134 Huang J, Yan L, Cheng Z, Wu H, Du L, Wang J, et al. A randomized trial comparing radiofrequency ablation and surgical resection for HCC conforming to the Milan criteria. Ann Surg. 2010 Dec;252(6):903-12.

135 Li L, Zhang J, Liu X, Li X, Jiao B, Kang T. Clinical outcomes of radiofrequency ablation and surgical resection for small hepatocellular carcinoma: a meta-analysis. J Gastroenterol Hepatol. 2012 Jan;27(1):51-8.

136 Hasegawa K, Aoki T, Ishizawa T, Kaneko J, Sakamoto Y, Sugawara Y, et al. Comparison of the therapeutic outcomes between surgical resection and percutaneous ablation for small hepatocellular carcinoma. Ann Surg Oncol. 2014 Jun;21(Suppl 3):S348-55.

137 Feng Q, Chi Y, Liu Y, Zhang L, Liu Q. Efficacy and safety of percutaneous radiofrequency ablation versus surgical resection for small hepatocellular carcinoma: a meta-analysis of 23 studies. J Cancer Res Clin Oncol. 2015 Jan; 141(1):1-9.

138 Morimoto M, Numata K, Kondou M, Nozaki A, Morita S, Tanaka K. Midterm outcomes in patients with intermediate-sized hepatocellular carcinoma: a randomized controlled trial for determining the efficacy of radiofrequency ablation combined with transcatheter arterial chemoembolization. Cancer. 2010 Dec 1;116(23): 5452-60.

139 Peng ZW, Zhang YJ, Chen MS, Xu L, Liang HH, Lin XJ, et al. Radiofrequency ablation with or without transcatheter arterial chemoembolization in the treatment of hepatocellular carcinoma: a prospective randomized trial. J Clin Oncol. 2013 Feb 1;31(4):426-32.

140 Chen QW, Ying HF, Gao S, Shen YH, Meng ZQ, Chen H, et al. Radiofrequency ablation plus chemoembolization versus radiofrequency ablation alone for hepatocellular carcinoma: a systematic review and meta-analysis. Clin Res Hepatol Gastroenterol. 2016 Jun;40(3):309-14.

141 Livraghi T, Meloni F, Di Stasi M, Rolle E, Solbiati L, Tinelli C, et al. Sustained complete response and complications rates after radiofrequency ablation of very early hepatocellular carcinoma in cirrhosis: is resection still the treatment of choice? Hepatology. 2008 Jan;47(1):82-9.

142 Peng ZW, Lin XJ, Zhang YJ, Liang HH, Guo RP, Shi M, et al. Radiofrequency ablation versus hepatic resection for the treatment of hepatocellular carcinomas $2 \mathrm{~cm}$ or smaller: a retrospective comparative study. Radiology. 2012 Mar;262(3):1022-33.

143 Kim YS, Lim HK, Rhim H, Lee MW, Choi D, Lee WJ, et al. Ten-year outcomes of percutaneous radiofrequency ablation as first-line therapy of early hepatocellular carcinoma: analysis of prognostic factors. J Hepatol. 2013 Jan;58(1):89-97.

144 Weis S, Franke A, Mössner J, Jakobsen JC, Schoppmeyer K. Radiofrequency (thermal) ablation versus no intervention or other interventions for hepatocellular carcinoma. Cochrane Database Syst Rev. 2013 Dec 19;(12): CD003046.

145 Zhang L, Ge NL, Chen Y, Xie XY, Yin X, Gan YH, et al. Long-term outcomes and prognostic analysis of radiofrequency ablation for small hepatocellular carcinoma: 10-year follow-up in Chinese patients. Med Oncol. 2015 Mar;32(3):77.

146 Cho YK, Kim JK, Kim MY, Rhim H, Han JK. Systematic review of randomized trials for hepatocellular carcinoma treated with percutaneous ablation therapies. Hepatology. 2009 Feb;49(2):453-9.

147 Shibata T, Iimuro Y, Yamamoto Y, Maetani Y, Ametani F, Itoh K, et al. Small hepatocellular carcinoma: comparison of radio-frequency ablation and percutaneous microwave coagulation therapy. Radiology. 2002 May;223(2):331-7.

148 Di Vece F, Tombesi P, Ermili F, Maraldi C, Sartori S. Coagulation areas produced by cool-tip radiofrequency ablation and microwave ablation using a device to decrease back-heating effects: a prospective pilot study. Cardiovasc Intervent Radiol. 2014 Jun;37(3):723-9.

149 Ahmed M, Solbiati L, Brace CL, Breen DJ, Callstrom MR, Charboneau JW, et al. Image-guided tumor ablation: standardization of terminology and reporting criteria: a 10 -year update. J Vasc Interv Radiol. 2014 Oct;25(11): 1691-e4.

150 Pelletier G, Ducreux M, Gay F, Luboinski M, Hagege H, Dao T, et al. Treatment of unresectable hepatocellular carcinoma with lipiodol chemoembolization: a multicenter randomized trial. Groupe CHC. J Hepatol. 1998 Jul; 29(1):129-34. http://dx.doi.org/10.1016/s0168-8278(98)80187-6.

151 Camma C, Schepis F, Orlando A, Albanese M, Shahied L, Trevisani F, et al. Transarterial chemoembolization for unresectable hepatocellular carcinoma: meta-analysis of randomized controlled trials. Radiology. 2002 Jul; 224(1):47-54. http://dx.doi.org/10.1148/radiol.2241011262.

152 Llovet JM, Real MI, Montaña X, Planas R, Coll S, Aponte J, et al. Arterial embolisation or chemoembolisation versus symptomatic treatment in patients with unresectable hepatocellular carcinoma: a randomised controlled trial. Lancet. 2002 May 18;359(9319):1734-9.

153 Lo CM, Ngan H, Tso WK, Liu CL, Lam CM, Poon RT, et al. Randomized controlled trial of transarterial lipiodol chemoembolization for unresectable hepatocellular carcinoma. Hepatology. 2002 May;35(5):1164-71. 
154 Llovet JM, Bruix J. Systematic review of randomized trials for unresectable hepatocellular carcinoma: chemoembolization improves survival. Hepatology. 2003 Feb;37(2):429-42.

155 Lencioni R, de Baere T, Soulen MC, Rilling WS, Geschwind JF. Lipiodol transarterial chemoembolization for hepatocellular carcinoma: a systematic review of efficacy and safety data. Hepatology. 2016 Jul;64(1):106-16.

156 Interventional Group of Chinese Society of Radiology.. Expert consensus for standard operating procedures for transcatheter arterial chemoembolization for primary hepatocellular carcinoma. Chin J Radiol. 2011; 45(10):908-12. http://dx.doi.org/10.3760/cma.j.issn.1005-1201.2011.10.003.

157 Yang M, Fang Z, Yan Z, Luo J, Liu L, Zhang W, et al. Transarterial chemoembolisation (TACE) combined with endovascular implantation of an iodine- 125 seed strand for the treatment of hepatocellular carcinoma with portal vein tumour thrombosis versus TACE alone: a two-arm, randomised clinical trial. J Cancer Res Clin Oncol. 2014 Feb;140(2):211-9.

$158 \mathrm{Hu}$ HT, Li HL, Guo CY, Yao QJ, Meng LY, Luo JP, et al. Transcatheter arterial chemoembolization combined 125iodine seed implantation for primary hepatic carcinoma with portal vein tumor thrombus thrombosis. Chin J Radiol. 2012;46(6). http://dx.doi.org/10.3760/cma.j.issn.1005-1201.2012.06.016.

159 Si ZM, Wang GZ, Qian S, Qu XD, Yan ZP, Liu R, et al. Combination therapies in the management of large ( $\geq 5 \mathrm{~cm})$ hepatocellular carcinoma: microwave ablation immediately followed by transarterial chemoembolization. J Vasc Interv Radiol. 2016 Oct;27(10):1577-83. http://dx.doi.org/10.1016/j.jvir.2016.02.014.

160 Jang JW, Choi JY, Bae SH, Kim CW, Yoon SK, Cho SH, et al. Transarterial chemo-lipiodolization can reactivate hepatitis B virus replication in patients with hepatocellular carcinoma. J Hepatol. 2004 Sep;41(3):427-35.

161 Bujold A, Massey CA, Kim JJ, Brierley J, Cho C, Wong RK, et al. Sequential phase I and II trials of stereotactic body radiotherapy for locally advanced hepatocellular carcinoma. J Clin Oncol. 2013 May 1;31(13):1631-9.

162 Wahl DR, Stenmark MH, Tao Y, Pollom EL, Caoili EM, Lawrence TS, et al. Outcomes after stereotactic body radiotherapy or radiofrequency ablation for hepatocellular carcinoma. J Clin Oncol. 2016 Feb 10;34(5):452-9.

163 Su TS, Liang P, Liang J, Lu HZ, Jiang HY, Cheng T, et al. Long-term survival analysis of stereotactic ablative radiotherapy versus liver resection for small hepatocellular carcinoma. Int J Radiat Oncol Biol Phys. 2017 Jul 1;98(3):639-46.

164 Chino F, Stephens SJ, Choi SS, Marin D, Kim CY, Morse MA, et al. The role of external beam radiotherapy in the treatment of hepatocellular cancer. Cancer. 2018 Sep 1;124(17):3476-89.

165 Hara K, Takeda A, Tsurugai Y, Saigusa Y, Sanuki N, Eriguchi T, et al. Radiotherapy for hepatocellular carcinoma results in comparable survival to radiofrequency ablation: a propensity score analysis. Hepatology. 2019 Jun; 69(6):2533-45.

166 Kim N, Kim HJ, Won JY, Kim DY, Han KH, Jung I, et al. Retrospective analysis of stereotactic body radiation therapy efficacy over radiofrequency ablation for hepatocellular carcinoma. Radiother Oncol. 2019 Feb; 131: 81-7.

167 Rim CH, Kim HJ, Seong J. Clinical feasibility and efficacy of stereotactic body radiotherapy for hepatocellular carcinoma: a systematic review and meta-analysis of observational studies. Radiother Oncol. 2019 Feb;131: 135-44.

168 Shen PC, Chang WC, Lo CH, Yang JF, Lee MS, Dai YH, et al. Comparison of stereotactic body radiation therapy and transarterial chemoembolization for unresectable medium-sized hepatocellular carcinoma. Int J Radiat Oncol Biol Phys. 2019 Oct 1;105(2):307-18.

169 Zeng ZC, Tang ZY, Fan J, Zhou J, Qin LX, Ye SL, et al. A comparison of chemoembolization combination with and without radiotherapy for unresectable hepatocellular carcinoma. Cancer J. 2004 Sep-0ct;10(5):307-16.

170 Zeng ZC, Fan J, Tang ZY, Zhou J, Qin LX, Wang JH, et al. A comparison of treatment combinations with and without radiotherapy for hepatocellular carcinoma with portal vein and/or inferiorvena cava tumor thrombus. Int J Radiat Oncol Biol Phys. 2005 Feb 1;61(2):432-43.

171 Meng MB, Cui YL, Lu Y, She B, Chen Y, Guan YS, et al. Transcatheter arterial chemoembolization in combination with radiotherapy for unresectable hepatocellular carcinoma: a systematic review and meta-analysis. Radiother Oncol. 2009 Aug;92(2):184-94.

172 Huo YR, Eslick GD. Transcatheter arterial chemoembolization plus radiotherapy compared with chemoembolization alone for hepatocellular carcinoma: a systematic review and meta-analysis. JAMA Oncol. 2015 Sep; 1(6):756-65.

173 Ohri N, Dawson LA, Krishnan S, Seong J, Cheng JC, Sarin SK, et al. Radiotherapy for hepatocellular carcinoma: new indications and directions for future study. J Natl Cancer Inst. 2016 Sep;108(9).

174 Shen L, Xi M, Zhao L, Zhang X, Wang X, Huang Z, et al. Combination therapy after TACE for hepatocellular carcinoma with macroscopic vascular invasion: stereotactic body radiotherapy versus sorafenib. Cancers. 2018 Dec 14;10(12).

175 Yoon SM, Ryoo BY, Lee SJ, Kim JH, Shin JH, An JH, et al. Efficacy and safety of transarterial chemoembolization plus external beam radiotherapy vs sorafenib in hepatocellular carcinoma with macroscopic vascular invasion: a randomized clinical trial. JAMA Oncol. 2018 May 1;4(5):661-9.

176 Jihye C, Jinsil S. Application of radiotherapeutic strategies in the BCLC-defined stages of hepatocellular carcinoma. Liver Cancer. 2012 Nov;1(3-4):216-25.

177 Soliman H, Ringash J, Jiang H, Singh K, Kim J, Dinniwell R, et al. Phase II trial of palliative radiotherapy for hepatocellular carcinoma and liver metastases. J Clin Oncol. 2013 Nov 1;31(31):3980-6. 


\begin{tabular}{l|l|}
\hline Liver Cancer 2020;9:682-720 \\
\hline DOI: 10.1159/000509424 & $\begin{array}{l}\text { @ 2020 The Author(s). Published by S. Karger AG, Basel } \\
\text { www.karger.com/lic }\end{array}$ \\
\hline
\end{tabular}

Zhou et al.: 2019 HCC Guidelines in China

178 Radiation Oncology Branch of the Chinese Medical Association; Expert Committee on Liver Cancer and Digestive System of China Institute of Biomedical Engineering; Liver Cancer Research Group of Radiology Oncology Branch of China Research Hospital.. Consensus on radiation therapy for primary liver cancer in 2016. Chin J Radiat Oncol. 2016;25(11):1141-50. http://dx.doi.org/10.3760/cma.j.issn.1004-4221.2016.11.001.

179 Wang WH, Wang Z, Wu JX, Zhang T, Rong WQ, Wang LM, et al. Survival benefit with IMRT following narrowmargin hepatectomy in patients with hepatocellular carcinoma close to major vessels. Liver Int. 2015 Dec; 35(12):2603-10.

180 Sapisochin G, Barry A, Doherty M, Fischer S, Goldaracena N, Rosales R, et al. Stereotactic body radiotherapy vs. TACE or RFA as a bridge to transplant in patients with hepatocellular carcinoma. An intention-to-treat analysis. J Hepatol. 2017 Jul;67(1):92-9.

181 Byun HK, Kim HJ, Im YR, Kim DY, Han KH, Seong J. Dose escalation by intensity modulated radiotherapy in liver-directed concurrent chemoradiotherapy for locally advanced BCLC stage C hepatocellular carcinoma. Radiother Oncol. 2019 Apr;133:1-8.

182 Zeng Z. Stereotactic body radiation therapy for hepatocellular carcinoma. Chinese J Oncol. 2015 Sep;37(9): 650-3. http://dx.doi.org/10.3760/cma.j.issn.0253-3766.2015.09.004.

183 Hu Y, Zhou YK, Chen YX, Shi SM, Zeng ZC. 4D-CT scans reveal reduced magnitude of respiratory liver motion achieved by different abdominal compression plate positions in patients with intrahepatic tumors undergoing helical tomotherapy. Med Phys. 2016 Jul;43(7):4335.

184 The Chinese Society of Nuclear Medicine Working Committee for Treatment of Bone Metastasis.. Expert consensus on strontium-89 chloride treatment of bone metastases (2017). Chin J Nucl Med Mol Imaging. 2018; 38(6):412-5. http://dx.doi.org/10.3760/cma.j.issn.2095-2848.2018.06.008.

185 Llovet JM, Ricci S, Mazzaferro V, Hilgard P, Gane E, Blanc JF, et al. Sorafenib in advanced hepatocellular carcinoma. N Engl J Med. 2008 Jul 24;359(4):378-90.

186 Cheng AL, Kang YK, Chen Z, Tsao CJ, Qin S, Kim JS, et al. Efficacy and safety of sorafenib in patients in the AsiaPacific region with advanced hepatocellular carcinoma: a phase III randomised, double-blind, placebocontrolled trial. Lancet Oncol. 2009 Jan;10(1):25-34.

187 Pressiani T, Boni C, Rimassa L, Labianca R, Fagiuoli S, Salvagni S, et al. Sorafenib in patients with Child-Pugh class A and B advanced hepatocellular carcinoma: a prospective feasibility analysis. Ann Oncol. 2013 Feb; 24(2):406-11.

188 Kudo M, Finn RS, Qin S, Han KH, Ikeda K, Piscaglia F, et al. Lenvatinib versus sorafenib in first-line treatment of patients with unresectable hepatocellular carcinoma: a randomised phase 3 non-inferiority trial. Lancet. 2018 Mar 24;391(10126):1163-73.

189 Qin S, Bai Y, Lim HY, Thongprasert S, Chao Y, Fan J, et al. Randomized, multicenter, open-label study of oxaliplatin plus fluorouracil/leucovorin versus doxorubicin as palliative chemotherapy in patients with advanced hepatocellular carcinoma from Asia. J Clin Oncol. 2013 Oct 1;31(28):3501-8.

190 Qin S, Cheng Y, Liang J, Shen L, Bai Y, Li J, et al. Efficacy and safety of the FOLFOX4 regimen versus doxorubicin in Chinese patients with advanced hepatocellular carcinoma: a subgroup analysis of the EACH study. Oncologist. 2014 Nov;19(11):1169-78.

191 Assenat E, Pageaux GP, Thézenas S, Peron JM, Becouarn Y, Seitz JF, et al. Sorafenib alone vs. sorafenib plus GEMOX as 1st-line treatment for advanced HCC: the phase II randomised PRODIGE 10 trial. Br J Cancer. 2019 Apr;120(9):896-902. http://dx.doi.org/10.1038/s41416-019-0443-4.

192 Qu FL, Hao XZ, Qin SK, Liu JW, Sui GJ, Chen Q, et al. Multicenter phase II clinical trial of arsenic trioxide injection in the treatment of primary hepatocarcinoma. Chinese J Oncol. 2011 Sep;33(9):697-701. http://dx.doi. org/10.3760/cma.j.issn.0253-3766.2011.09.013.

193 Bruix J, Qin S, Merle P, Granito A, Huang YH, Bodoky G, et al. Regorafenib for patients with hepatocellular carcinoma who progressed on sorafenib treatment (RESORCE): a randomised, double-blind, placebocontrolled, phase 3 trial. Lancet. 2017 Jan 7;389(10064):56-66.

194 El-Khoueiry AB, Sangro B, Yau T, Crocenzi TS, Kudo M, Hsu C, et al. Nivolumab in patients with advanced hepatocellular carcinoma (CheckMate 040): an open-label, non-comparative, phase 1/2 dose escalation and expansion trial. Lancet. 2017 Jun 24;389(10088):2492-502.

195 Zhu AX, Finn RS, Edeline J, Cattan S, Ogasawara S, Palmer D, et al. Pembrolizumab in patients with advanced hepatocellular carcinoma previously treated with sorafenib (KEYNOTE-224): a non-randomised, open-label phase 2 trial. Lancet Oncol. 2018 Jul;19(7):940-52. http://dx.doi.org/10.1016/S1470-2045(18)30351-6.

196 Puzanov I, Diab A, Abdallah K, Bingham CO 3rd, Brogdon C, Dadu R, et al. Managing toxicities associated with immune checkpoint inhibitors: consensus recommendations from the society for immunotherapy of cancer (SITC) toxicity management working group. J Immunother Cancer. 2017 Nov 21;5(1):95.

197 Brahmer JR, Lacchetti C, Schneider BJ, Atkins MB, Brassil KJ, Caterino JM, et al. Management of immune-related adverse events in patients treated with immune checkpoint inhibitor therapy: American society of clinical oncology clinical practice guideline. J Clin Oncol. 2018 Jun 10;36(17):1714-68.

198 Haanen JBAG, Carbonnel F, Robert C, Kerr KM, Peters S, Larkin J, et al. Management of toxicities from immunotherapy: ESMO clinical practice guidelines for diagnosis, treatment and follow-up. Ann Oncol. 2018 Oct 1; 29(Suppl 4):iv264-66.

199 Cheng SQ, Wu MC, Chen H, Shen F, Yang JH, Zhao YX, et al. Anti-recurrence effects of thymosin $\alpha 1$ in patients with primary liver cancer after hepatectomy. Chin J Hepatobiliary Surg. 2004;10(9):592-3. http://dx.doi. org/10.3760/cma.j.issn.1007-8118.2004.09.006. 
200 Wang Y, Chen M, Wu Z, Tong C, Dai H, Guo Y, et al. CD133-directed CAR T cells for advanced metastasis malignancies: a phase I trial. Oncoimmunology. 2018;7(7):e1440169.

201 Lee JH, Lee JH, Lim YS, Yeon JE, Song TJ, Yu SJ, et al. Adjuvant immunotherapy with autologous cytokineinduced killer cells for hepatocellular carcinoma. Gastroenterology. 2015 Jun; 148(7):1383.

202 Abou-Alfa GK, Meyer T, Cheng AL, El-Khoueiry AB, Rimassa L, Ryoo BY, et al. Cabozantinib in patients with advanced and progressing hepatocellular carcinoma. N Engl J Med. 2018 Jul 5;379(1):54-63.

203 Zhu AX, Park JO, Ryoo BY, Yen CJ, Poon R, Pastorelli D, et al. Ramucirumab versus placebo as second-line treatment in patients with advanced hepatocellular carcinoma following first-line therapy with sorafenib (REACH): a randomised, double-blind, multicentre, phase 3 trial. Lancet Oncol. 2015 Jul;16(7):859-70.

204 Zhu AX, Kang YK, Yen CJ, Finn RS, Galle PR, Llovet JM, et al. Ramucirumab after sorafenib in patients with advanced hepatocellular carcinoma and increased $\alpha$-fetoprotein concentrations (REACH-2): a randomised, double-blind, placebo-controlled, phase 3 trial. Lancet Oncol. 2019 Feb;20(2):282-96. http://dx.doi. org/10.1016/S1470-2045(18)30937-9.

205 China Association of Chinese Medicine.. Traditional Chinese medicine guidelines for diagnosis and treatment of cancer. Beijing: China Press of Traditional Chinese Medicine; 2008.

206 Yan T, Bi XY, Fang Y, Zhou JG, Zhao JJ, Huang Z, et al. Adiuvant huaier on long-term survivals after curative hepatectomy for primary liver cancer. Chin J Hepatobiliary Surg. 2012;18(2):99-102. http://dx.doi. org/10.3760/cma.j.issn.1007-8118.2012.02.006.

207 Peng WD. Clinical observation on the effect of Fufangbanmaocapsules combined with chemotherapy for advanced liver cancer. Anti-Tumor Pharmacy. 2011;1(06):518-9+43. http://dx.doi.org/10.3969/j.issn.20951264.2011.06.013

208 Gao JL. Prospective randomized controlled study on advanced primary hepatic cancer treated by Ganfule prescription. China J Chinese Materia Medica. 2014;39(12):2367-9. http://dx.doi.org/10.4268/ cjcmm20141243.

209 Cheng Y, Hua HQ. Research progress on anti-hepatoma mechanisms and clinical application of $\beta$-elemene. Chin Clin Oncol. 2017;22(10):950-3. http://dx.doi.org/10.3969/j.issn.1009-0460.2017.10.018.

210 Lu DP, Wang YQ, Zhao WL, Qi ZP. A clinical study of Kanglaite in combination with TACE for treatment of liver cancer. World Clin Med. 2017;11(5):70-2.

211 Yang XH. Effect of TACE in combination with intravenous infusion of brucea javanica oil emulsion on clinical efficacy and VEGF level in patients with liver cancer. Strait Pharm J. 2017;29(09):176-7. http://dx.doi. org/10.3969/j.issn.1006-3765.2017.09.090.

212 Cai W, Yin C, Fan Q. An clinical observation of Aidi in combination with transcatheter arterial chemoembolization for treatment of mid-advanced primary liver cancer. J Chin Physicians. 2018;20(11):1723-5. http:// dx.doi.org/10.3760/cma.j.issn.1008-1372.2018.11.032.

213 Fan S, Li QY, Zhou ZT, He WL, Wu ZG, Zhong P. A study of efficacy of TACE in combination with Jinlong capsules for treatment of primary liver cancer. China Pract Med. 2019;14(21):42-4. http://dx.doi.org/10.14163/j. cnki.11-5547/r.2019.21.022.

214 Chinese Society of Infectious Diseases, Chinese Medical Association; Chinese Society of Hepatology, Chinese Medical Association.. Guidelines for the prevention and treatment of chronic hepatitis B (version 2019). I Clin Hepatol. 2019;35(12):2648-69. http://dx.doi.org/10.3969/j.issn.1001-5256.2019.12.007.

215 European Association for the Study of the Liver. Electronic address EEE, European Association for the Study of the L; European Association for the Study of the Liver.. EASL recommendations on treatment of hepatitis C 2018. J Hepatol. 2018 Aug;69(2):461-511. http://dx.doi.org/10.1016/j.jhep.2018.03.026.

216 Chinese Society of Hepatology, Chinese Medical Association; Chinese Society of Infectious Diseases, Chinese Medical Association.. Guidelines for the prevention and treatment of hepatitis C (2019 version). J Clin Hepatol. 2019;35(12):2670-86. http://dx.doi.org/10.3969/j.issn.1001-5256.2019.12.008.

217 Seymour L, Bogaerts J, Perrone A, Ford R, Schwartz LH, Mandrekar S, et al. iRECIST: guidelines for response criteria for use in trials testing immunotherapeutics. Lancet Oncol. 2017 Mar;18(3):e143-52.

218 Yoshida H, Mamada Y, Taniai N, Uchida E. Spontaneous ruptured hepatocellular carcinoma. Hepatol Res. 2016 Jan;46(1):13-21.

219 Zhong F, Cheng XS, He K, Sun SB, Zhou J, Chen HM. Treatment outcomes of spontaneous rupture of hepatocellular carcinoma with hemorrhagic shock: a multicenter study. Springerplus. 2016;5(1):1101.

220 Moris D, Chakedis J, Sun SH, Spolverato G, Tsilimigras DI, Ntanasis-Stathopoulos I, etal. Management, outcomes, and prognostic factors of ruptured hepatocellular carcinoma: a systematic review. J Surg Oncol. 2018 Mar; 117(3):341-53.

221 Sahu SK, Chawla YK, Dhiman RK, Singh V, Duseja A, Taneja S, et al. Rupture of hepatocellular carcinoma: a review of literature. J Clin Exp Hepatol. 2019 Mar-Apr;9(2):245-56.

222 Lai EC, Lau WY. Spontaneous rupture of hepatocellular carcinoma: a systematic review. Arch Surg. 2006 Feb; 141(2):191-8.

223 Aoki T, Kokudo N, Matsuyama Y, Izumi N, Ichida T, Kudo M, et al. Prognostic impact of spontaneous tumor rupture in patients with hepatocellular carcinoma: an analysis of 1160 cases from a nationwide survey. Ann Surg. 2014 Mar;259(3):532-42.

224 Shin BS, Park MH, Jeon GS. Outcome and prognostic factors of spontaneous ruptured hepatocellular carcinoma treated with transarterial embolization. Acta Radiol. 2011 Apr 1;52(3):331-5. 